\title{
Risk Trading in Energy Communities
}

\author{
Vespermann, Niklas; Hamacher, Thomas; Kazempour, Jalal
}

\section{Published in:}

IEEE Transactions on Smart Grid

Link to article, DOI:

10.1109/TSG.2020.3030319

Publication date:

2021

Document Version

Peer reviewed version

Link back to DTU Orbit

Citation (APA):

Vespermann, N., Hamacher, T., \& Kazempour, J. (2021). Risk Trading in Energy Communities. IEEE Transactions on Smart Grid, 12(2), 1249 - 1263. https://doi.org/10.1109/TSG.2020.3030319

\section{General rights}

Copyright and moral rights for the publications made accessible in the public portal are retained by the authors and/or other copyright owners and it is a condition of accessing publications that users recognise and abide by the legal requirements associated with these rights.

- Users may download and print one copy of any publication from the public portal for the purpose of private study or research.

- You may not further distribute the material or use it for any profit-making activity or commercial gain

- You may freely distribute the URL identifying the publication in the public portal

If you believe that this document breaches copyright please contact us providing details, and we will remove access to the work immediately and investigate your claim. 


\title{
Risk Trading in Energy Communities
}

\author{
Niklas Vespermann, Student Member, IEEE, Thomas Hamacher, and Jalal Kazempour, Senior Member, IEEE
}

\begin{abstract}
Local energy communities are proposed as a regulatory framework to enable the market participation of endconsumers. However, volatile local market-clearing prices, and consequently, volatile cost give rise to local market participants with generally heterogeneous risk attitudes. To prevent the increased operational cost of communities due to conservative trading decisions in the forward stage, e.g., a day-ahead market, we propose risk trading in energy communities via financial hedging products, the so-called Arrow-Debreu securities. The conditional value-at-risk serves as our risk measure for players to study different degrees of market completeness for risk. We define a risk-averse Nash game with risk trading and solve the Nash equilibrium problem for an incomplete market for risk as a mixed complementarity problem. We show that such a Nash equilibrium problem reduces to a single optimization problem if the market is complete for risk. Numerical findings indicate that a significant community cost saving can be realized when players engage in risk trading and sufficient financial hedging products are available. Moreover, risk trading efficiently protects less risk-averse players from highly risk-averse decision-making of rival players.
\end{abstract}

Index Terms-Arrow-Debreu security, conditional value-atrisk, energy community, market completeness for risk, mixed complementarity problem, risk trading, two-stage stochastic Nash equilibrium problem

\section{INTRODUCTION}

\section{A. Motivation and Aim}

The development of distributed energy resources and the progress in information and communication technologies empower end-consumers to engage in energy trading. Local energy communities are proposed as a regulatory framework that allows the market participation of these proactive consumers, the so-called prosumers [1]-[3]. Energy trading within a local energy community enables its members to efficiently utilize distributed energy resources, such as roof-top photovoltaic (PV) systems, battery storage units, and thermalelectric appliances. In this way, community members are expected to reduce their energy cost [4].

However, the uncertainty inherent to the intermittent injection of small-scale renewable energy sources such as PV systems causes increased price variability within the energy community. As a result, the volatility of energy cost for local energy market participants, the so-called players, increases.

Generally, energy markets are organized in a temporally sequential manner to ensure a cost-efficient matching of supply

N. Vespermann, T. Hamacher are with the Department of Electrical and Computer Engineering, Technical University of Munich, Germany, e-mail: \{niklas.vespermann, thomas.hamacher\}@tum.de.

J. Kazempour is with the Department of Electrical Engineering, Technical University of Denmark, 2800 Kgs. Lyngby, Denmark, e-mail: seykaz@elektro.dtu.dk.

Manuscript received March 26, 2020; revised July 27, 2020; accepted September 29, 2020. Date of publication [...] and demand given the physical requirements of the technical system, such as the instantaneous balance of electricity production and consumption. In this context, players make decisions in a forward market, e.g., a day-ahead market, before the realization of an uncertain event. The potential realizations of the uncertainty in a spot market, e.g., a realtime market, induce volatile prices, i.e., the spot market price might be comparatively higher or lower than the forward market price depending on the realization of the uncertain event. This price volatility leads to a cost volatility for players, giving rise to risk-averse preferences. ${ }^{1}$ At the stage before uncertainty realization, i.e., the forward market, a risk-averse player tends to make conservative decisions such that the volatility of her overall cost is reduced in both markets. This risk-averse decision-making comes with the cost of an increased disbenefit, i.e., a higher cost or a lower profit for individual players as well as a higher operational cost for the whole energy community.

Under the common assumption of homogeneous risk aversion [6], all players have identical risk attitudes. However, in practice we rather observe heterogeneous risk aversion, where individual players have different risk preferences, such that some players are willing to accept a greater cost volatility induced by an uncertain event, e.g., the power generation of renewable energy sources, and thereby, a greater risk than others. Consequently, if less risk-averse players could take over the cost volatility of highly risk-averse players, these highly risk-averse players are able to make less conservative forward market decisions while ensuring a low cost volatility. As a result, the total cost of the energy community as well as disbenefits of risk-averse players resulting from conservative forward market decisions decrease.

To this purpose, we propose risk trading within local energy communities as a vehicle for the cost volatility transfer. Risk trading describes the exchange of financial hedging products in a market to reduce the cost volatility by a different mean than conservative forward market decisions. However, this transfer depends highly on the availability of financial hedging products. In this context, local energy markets might range from a fully incomplete market for risk, where no financial hedging products are available, to a complete market for risk [7], in which all potential realizations of the uncertainty can be hedged. Both degrees of market completeness for risk constitute extremes, while intermediate cases are those in which risk trading is possible only for a part of potential

\footnotetext{
${ }^{1}$ Risk aversion is a natural attitude of decision-makers, which can be observed not only in long-term planning decision-making problems but also in short-term operational ones. However, since operational decisions are made more often, e.g., on an hourly basis, decision-makers are able to adjust their risk preferences over time. They can even learn how to play in dynamic games with risky payoffs [5].
} 
realizations of the uncertainty.

The concept of risk trading gives rise to two important market-driven questions: First, how to define and implement a financial hedging product for energy communities to transfer the cost volatility from highly risk-averse to less risk-averse players? Second, does a potentially unique market equilibrium under heterogeneous risk aversion and risk trading exist?

To answer these questions, we consider a two-stage stochastic decision-making process for heterogeneously risk-averse energy community members, who engage in risk trading. The first stage corresponds to trading decisions in a local forward market for energy and financial hedging products. The second stage represents trading decisions in a local spot market, where the uncertain PV power generation is characterized through a set of discrete scenarios. We apply the well-known Conditional Value-at-Risk (CVaR) [8] as the risk measure for players, and then define a risk-averse non-cooperative Nash game with risk trading based on the collection of all players' decision-making problems. Next, we solve the resulting two-stage stochastic Nash equilibrium problem as a mixed complementarity problem [9]. However, for the special case of a complete market for risk, we solve a single two-stage stochastic optimization problem, which is equivalent to the Nash equilibrium problem. We eventually evaluate different degrees of market completeness for risk in terms of individual costs and total community cost.

\section{B. Literature Review}

Neglecting risk aversion causes a systematic overestimation of benefits as shown in [10], where heterogeneously riskaverse players are considered for a generation expansion planning problem. Reference [11] discusses the effect of different risk preferences on market-clearing outcomes. Riskaverse stochastic producers avoid the forward market, which causes the system cost and market-clearing prices to increase. However, if risk trading among players is available, the cost volatility can be shifted through risk trading to less riskaverse players as discussed in [12]. Thereby, even highly riskaverse players make moderate forward market decisions, since the volatile cost is hedged. Thus, market-clearing prices and the system cost decrease. Reference [13] even highlights the potential benefits of risk trading in the case of ill-designed electricity markets.

From a methodological perspective, [14] and [15] link heterogeneous risk aversion and complete as well as incomplete markets for risk by stating the problem as a risk-averse Nash equilibrium problem with risk trading. If the market is incomplete for risk, multiple equilibria may exist [16], which are differently stable and may have different system cost as discussed in [17]. However, if the market is complete for risk, [14], [15], and [18] show how a risk-averse social planner solution can be interpreted as a perfectly competitive riskaverse Nash equilibrium, where sufficient hedging products are available.

The principles for market clearing and risk trading in an energy community are not different than those in other electricity markets, e.g., the transmission-level wholesale markets. However, since the number of players in local energy markets is quite limited, their heterogeneous risk preferences potentially have a great impact on market outcomes. In this context of heterogeneous risk aversion in energy communities the recent work [19] addresses the impact of risk trading on the notion of fairness among community members. Moreover, [20] studies heterogeneous risk-averse prosumers, where risk trading is based on a distributed implementation of financial products. A risk-averse equilibrium is computed given a generalized potential game structure.

However, a great challenge remains in defining an applicable financial product that enables risk trading. Here, [21] studies a local energy market on the distribution system level, i.e., a market organization layer above energy communities, where financial hedging rights are proposed to reduce the price volatility due to distribution network constraints. Moreover, [22] considers the simultaneous trading of energy and the uncertain part of power generation by PV systems. Note that financial products proposed by [21] and [22] leave the market partially incomplete for risk [23].

\section{Contributions and Paper Organization}

To the best of our knowledge, there are only two works in the existing literature that account for heterogeneously riskaverse players in the context of local energy communities. While [19] focuses on the notion of fairness, our work places strong emphasis on game-theoretical analyses and properties. In addition, [20] studies the risk-averse equilibrium based on a generalized Nash equilibrium problem, while we provide a formulation which yields a Nash equilibrium problem, and therefore rigorous conclusions on the solution existence and uniqueness. Moreover, [19] and [20] neglect a thorough analysis of different degrees of market completeness for risk.

Methodologically, we move beyond [14] and [15] and mathematically prove that no equivalent optimization problem necessarily exists in the case that the market is incomplete for risk, while the degree of incompleteness-either fully or partially-is arbitrary. Moreover, in contrast to [14], [15], and [18], we show that although for the complete case an equivalent optimization problem exists, multiple Nash equilibria might still be found.

From the application perspective, we offer a thorough study on local energy communities with a focus on risk-averse prosumers, who engage in risk trading. Based on our numerical results, we identify that prosumers with a deterministic demand avoid the uncertain spot market, while prosumers with stochastic generation tend to postpone trading decisions to the spot market, where the realization of the uncertain power generation is observed. Moreover, by risk trading a significant community cost saving can be realized, while all players yield reduced disbenefits.

The remainder of this paper is organized as follows. In Section II we introduce the structure of the energy community and describe methods for representing risk aversion and risk trading. We present risk-neutral optimization problems and start by defining a risk-neutral Nash game in Section III. In Section IV we extend risk-neutral optimization problems by adding the CVaR as a risk measure for players as well as 
risk trading among energy community members and define the risk-averse Nash game with risk trading. In Section V we present and discuss numerical results. We conclude our work in Section VI. Mathematical proofs of propositions are provided in Appendix A. Appendix B includes the resulting mixed complementarity problem. Lastly, Appendix $C$ provides the formulation for a risk-averse social planner optimization problem whose solution is equivalent to a risk-averse Nash game in the case that the market is complete for risk.

\section{PRELIMINARIES}

\section{A. Energy Communities}

We understand a local energy community as an aggregation of a few prosumers, which are spatially located very close to each other and physically connected to the distribution system as a single entity. For example, energy communities were recently demonstrated through "The Energy Collective" project [24] implemented in Bofællesskabet Svalin, Denmark, the "EnergyLab Nordhavn" project [25] implemented in the Nordhavn neighborhood of Copenhagen, Denmark, as well as the "pebbles" project [26] implemented in Wildpoldsried, Germany. These projects enable prosumers to directly engage in energy trading with their neighbors via a local energy market within the energy community. This is one key aspect that the European Commission asks its member states for by the renewable energy directive, Article 21 [27].

Local markets for energy communities should be differentiated from any other market schemes on the distribution system level that may geographically cover a whole feeder or even a suburb. Distribution-level markets might be designed for trading energy [28] or flexibility [29], taking into account power losses and technical constraints such as limits on nodal voltage magnitudes and apparent power flow of lines. We treat local markets for energy communities as one marketorganization layer below any distribution-level market, and rather see the energy community as a whole as a potential market participant in such distribution-level markets. However, we leave the explicit consideration for future research.

\section{B. Definitions}

In this study we consider a local forward as well as a local spot market, and represent the probability distribution of an uncertain realization in the spot market by a finite number of discrete scenarios $\omega \in \Omega$. Each scenario embodies a collection of the PV power generation for all prosumers under that scenario. Prior to the uncertainty realization, the local forward market determines the energy production and consumption schedule for each community member for, e.g., the next day, as well as a local forward market-clearing price. In real time when the uncertainty is realized, any deviations from the local forward market schedule are balanced in the local spot market, providing a local spot market-clearing price.

In the local forward market, risk-neutral players make decisions based on physical probabilities $\pi_{\omega}^{\Theta}$ of uncertain realizations, i.e., empirical real-world observations [15]. Riskaverse players, however, observe physical probabilities and

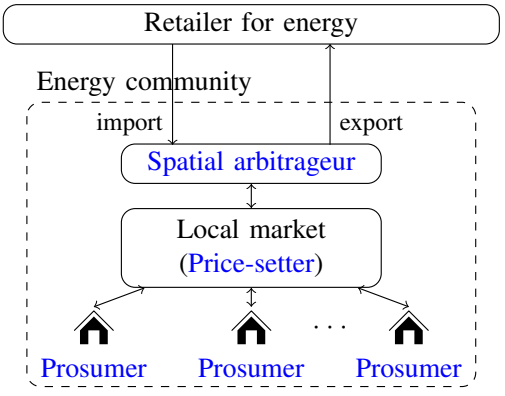

(a)

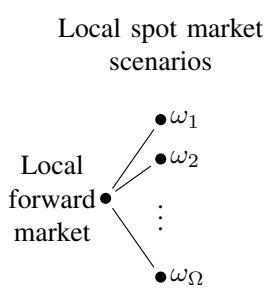

(b)
Fig. 1: Plot 1a shows the structure of the energy community, where explicitly considered players are marked in blue. Plot $1 \mathrm{~b}$ depicts the two-stage stochastic decision-making process of energy community members.

increase/decrease the weight of some scenarios to derive riskadjusted probabilities $\pi_{\omega}^{\rho}$ [10], which describe their individual risk preferences on uncertain realizations. In fact, a risk-averse player treats risk-adjusted probabilities as her individual decision variables, such that she considers comparatively higher weights for scenarios under which she incurs a high cost, or achieves a low profit. Similarly, she considers comparatively lower weights for scenarios, resulting in a low cost, or a high profit. Note that the sum of risk-adjusted probabilities should still be equal to one. By decision-making based on resulting risk-adjusted probabilities, the risk-averse player reduces her cost volatility at the cost of increased disbenefits.

To outweigh conservative forward market decisions and to reach a consensus among energy community members on risk preferences, we consider risk trading via a financial market product, the so-called Arrow-Debreu security [30]. ${ }^{2}$ For each scenario $\omega \in \Omega$, an Arrow-Debreu security is an unconstrained contract between a security buyer and a seller in the forward market. Both the buyer and seller are local energy community members. The buyer pays to the seller in the forward market based on a market-driven price, the socalled risk price $\mu_{\omega}$, whose value is lower than or equal to 1 . In return, the buyer receives from the seller a payment of 1 in the spot market if scenario $\omega$ realizes. For example, if a prosumer buys 100 securities from another prosumer for a given scenario $\omega$, she should pay $€ 100 \mu_{\omega}$ but will be paid back $€ 100$ if that scenario occurs. We will show later in Section $\mathrm{V}$ how risk-adjusted probabilities converge towards a consensus on risk preferences if risk trading is possible.

\section{Local Market Structure}

Within the energy community we explicitly consider three types of players as illustrated in Figure 1a, namely prosumers, a spatial arbitrageur and a price setter. Moreover, Figure 1b schematically depicts the underlying two-stage stochastic decision-making process of each player.

Prosumers, who potentially have an inelastic demand and own PV systems with an uncertain power generation, buy and sell energy as well as Arrow-Debreu securities in the

\footnotetext{
${ }^{2}$ While the definition of Arrow-Debreu securities in [30] is based on a discretization of an uncertain event, recent efforts have been made to consider risk trading for a continuous probability distribution of an uncertain event [31].
} 
local forward market. At the same time, they anticipate all possible local spot market realizations $\omega \in \Omega$, i.e., the spot market-clearing price and the uncertain PV power generation in scenario $\omega$, as well as their respective response in terms of energy trades and the cost/revenue from buying/selling ArrowDebreu securities for each scenario $\omega$.

In the case of a shortage or a surplus of energy the spatial arbitrageur imports and exports energy from and to the energy community. Since an energy community supplies and demands fairly small energy volumes, a direct access for the spatial arbitrageur to the wholesale market is rather unlikely. Therefore, we consider a retailer ${ }^{3}$, who serves as an intermediary for the energy community. The spatial arbitrageur decides on energy imports and exports as well as her Arrow-Debreu security trades in the local forward market. She also takes into account all possible realizations of local spot market-clearing prices as well as her optimal recourse in terms of energy imports and exports and the cost/revenue from Arrow-Debreu securities for each scenario $\omega$.

Finally, a price setter, who is a fictitious player [32], reveals local market-clearing prices evolving under free trade and perfect competition. In detail, the price setter reveals a local forward market-clearing price for energy and prices for ArrowDebreu securities given trading decisions by prosumers and energy imports/exports by the spatial arbitrageur. At the same time, she anticipates the recourse by prosumers due to the uncertain PV power generation as well as the optimal response by the spatial arbitrageur and reveals a spot market-clearing price for each scenario $\omega \in \Omega .{ }^{4}$

\section{Towards a Complete Market for Risk}

We study four different cases as summarized in Table I. In a case wherein all players are risk-neutral, a Nash equilibrium is determined by an optimization problem. The fully incomplete case constitutes a setting where prosumers and the spatial arbitrageur are heterogeneously risk-averse and no financial hedging products are available. We relax the constraint on the availability of hedging products and consider a partially incomplete market for risk, where some realizations of the uncertain PV power generation can be hedged. For these two cases of an incomplete market for risk, we rely on solving the Nash equilibrium problem as a mixed complementarity problem along with its challenges [16], [17], such as potential multiplicity, instability, and computational burden. However, if the market is complete for risk, i.e., all realizations of uncertain PV power generation can be hedged, the Nash equilibrium problem reduces to an equivalent optimization problem [18]. According to [15], expected disbenefits decrease as we move from a fully incomplete to a complete market for risk, as illustrated in the last column of Table I.

\footnotetext{
${ }^{3} \mathrm{~A}$ retailer is a self-interested profit-seeking entity which buys great energy volumes at the wholesale market and sells energy to many small customers.

${ }^{4}$ In a perfectly competitive market environment the spatial arbitrageur and the price setter can be interpreted as a community manager, who simultaneously fulfills both tasks, ensuring liquidity and revealing prices [9].
}

TABLE I: Problem overview with respect to the degree of risk trading.

\begin{tabular}{llll}
\hline Risk attitude & Risk trading & Problem type & Expected disbenefits \\
\hline Neutral & - & Optimization* $^{*}$ & $\star$ \\
Averse & Fully incomplete & Equilibrium $^{+}$ & $\star \star \star \star$ \\
Averse & Partially incomplete & Equilibrium $^{+}$ & $\star \star \star$ \\
Averse & Complete & Optimization* $^{*}$ & $\star \star$ \\
\hline${ }^{*}$ Equivalent & Nash equilibrium problem exists.
\end{tabular}

${ }^{+}$Equivalent optimization problem does not necessarily exist.

\section{E. Overview of the Conditional Value-at-Risk}

The seminal work [33] defines coherent risk measures $\rho(\cdot)$ of an uncertain disbenefit function $Z_{\omega}$. We will apply the dual representation of a coherent risk measure defined by [34] as

$$
\rho\left(Z_{\omega}\right)=\max _{\pi_{\omega}^{\rho} \in \mathcal{D}} \sum_{\omega \in \Omega} \pi_{\omega}^{\rho} Z_{\omega}
$$

where the risk set $\mathcal{D}$ defines the feasible region for riskadjusted probabilities $\pi_{\omega}^{\rho}$. For the $\mathrm{CVaR}$ as a coherent risk measure in particular, the risk set $\mathcal{D}^{\mathrm{CVaR}}$ [10] is given by

$$
\mathcal{D}^{\mathrm{CVaR}}=\left\{\pi_{\omega}^{\rho}: \sum_{\omega \in \Omega} \pi_{\omega}^{\rho}=1,0 \leq \pi_{\omega}^{\rho} \leq \frac{1}{\alpha} \pi_{\omega}^{\Theta}\right\},
$$

where the equality constraint ensures that the sum of riskadjusted probabilities is still equal to one. The lower bound of the inequality constraint ensures that all risk-adjusted probabilities $\pi_{\omega}^{\rho}$ are non-negative. The upper bound allows increasing the weight of some scenarios according to physical probabilities $\pi_{\omega}^{\Theta}$ and the risk aversion parameter $\alpha \in(0,1]$ indicating the percentile of the CVaR measure. For the special case $\alpha=1$, risk-adjusted probabilities are equal to physical probabilities, which represents a risk-neutral attitude.

In fact, (1a) with the risk set (1b) states a constrained optimization problem that determines risk-adjusted probabilities according to the risk aversion expressed by the CVaR. This optimization problem is an equivalent representation of the CVaR metric expressed by the well-known linear programming problem [8] as

$$
\rho^{\mathrm{CVaR}}\left(Z_{\omega}\right)=\min _{\zeta}\left\{\zeta+\frac{1}{\alpha} \sum_{\omega \in \Omega} \pi_{\omega}^{\Theta}\left(Z_{\omega}-\zeta\right)^{+}\right\},
$$

which states the weighted mean deviation from the $\alpha^{\text {th }}$ quantile, where $\zeta$ denotes the value-at-risk. However, implementing the CVaR by (1c) causes the objective function to be nonsmooth, i.e., non-continuously differentiable, owning to the positively defined term in $(\cdot)^{+}$, and thus, limits our possibilities for deriving optimality conditions to draw conclusions on the uniqueness of the equilibrium point in game-theoretic models.

Remark 1. In Section IV we combine the dual representation of a coherent risk measure (1a) with the risk set of the CVaR (1b) when introducing the generic framework of a riskaverse Nash equilibrium problem with risk trading. This allows us to use a framework that relies on analyzing the resulting game-theoretic models in their equivalent forms of variational inequality (VI) problems [35]. This VI representation enables us to draw conclusions on the existence and uniqueness of a game solution. For the case of a complete market for risk, 
we apply the CVaR metric (1c) in the equivalent optimization problem of the Nash equilibrium problem. This allows us to efficiently solve the equivalent optimization problem and derive numerical values of trades and prices.

\section{F. Assumptions}

We assume that all players are price takers, resulting in a perfectly competitive local energy market. The PV power generation is the only source of uncertainty, though other sources of uncertainty can be incorporated in the same manner. Owning to the low spatial distance, we assume that all PV systems have the same power generation profile and players possess the same information on scenarios as well as identical beliefs on physical probabilities $\pi_{\omega}^{\Theta}$. ${ }^{5}$ Furthermore, owning to the size of the energy community and the fact that spatial distances and trade volumes are relatively small, we neglect network constraints within the energy community. However, network constraints between the energy community and the distribution system, which are observed by the spatial arbitrageur are of interest and are modeled in this work. Results change in a way that a network congestion and consequently limited energy imports or exports affect local forward and spot market-clearing prices. Moreover, we consider the local market clearing for a single hour only, since we do not consider any technology with time-coupling constraints, e.g., energy storage units. A problem extension to consider multiple time steps is mathematically straightforward, though it complicates the solution interpretation on how risk trading affects local energy market outcomes. Lastly, we consider two trading floors, e.g., day-ahead and real-time, only, and exclude additional floors, such as intraday markets. The possibility of peerto-peer trading among prosumers within the community is also discarded.

In the next two sections we introduce the mathematical formulation of each player's problem. We start by describing a risk-neutral energy community in Section III. In Section IV, we extend the risk-neutral problem formulation to a setting with risk-averse players and risk trading.

Notation: We use a tilde, i.e., (.), for those symbols associated with the local spot market. Symbols followed by a colon denote dual variables of respective constraints. We use these dual variables when we derive Karush-Kuhn-Tucker (KKT) conditions of optimization problems in Appendix B.

\section{Market Clearing with Risk-Neutral Players}

In a risk-neutral setting, each prosumer $n \in \mathcal{P}$ minimizes her expected energy cost $J_{n}$ of meeting the demand as



$$
\begin{aligned}
& \text { s.t. } p_{n}+\tilde{p}_{n \omega}+\tilde{S}_{n \omega}-D_{n}=0: \tilde{\phi}_{n \omega}, \forall \omega \text {, } \\
& -\bar{P}_{n} \leq p_{n} \leq \bar{P}_{n}: \underline{\chi}_{n}^{\mathrm{p}}, \bar{\chi}_{n}^{\mathrm{p}}, \\
& \left.-\bar{P}_{n} \leq \tilde{p}_{n \omega} \leq \bar{P}_{n}: \underline{\chi}_{n \omega}^{\tilde{\mathrm{p}}}, \bar{\chi}_{n \omega}^{\tilde{\mathrm{p}}}, \forall \omega\right\} \forall n \text {. }
\end{aligned}
$$

${ }^{5} \mathrm{~A}$ relevant model accounting for asymmetric information about scenarios, but without modeling risk aversion, is available in [36].
The first term in the objective function (2a) states the cost incurred from power trades in the local forward market. Positive values of $p_{n}$ indicate a demand while negative values state a supply. Each prosumer $n$ pays/is paid based on the local forward market-clearing price $\lambda$ for her energy exchange $p_{n}$. The second term in (2a) states a regularizer [37] for forward market trades, where $\beta$ is a small positive constant ${ }^{6}$, e.g., $10^{-3}$. Institutionally, this regularizer can be interpreted as a transaction cost arising from trades. The third and fourth terms of (2a) refer to the expected cost and the regularizer in the local spot market, weighted by physical probabilities $\pi_{\omega}^{\Theta}$. The prosumer $n$ pays/is paid based on the local spot marketclearing price $\tilde{\lambda}_{\omega}$ for her power exchange $\tilde{p}_{n \omega}$ in scenario $\omega$. Note that market-clearing prices $\lambda$ and $\tilde{\lambda}_{\omega}$ are parameters within (2), while they are variables in the Nash equilibrium problem, i.e., the collection of all prosumers' $n \in \mathcal{P}$, the spatial arbitrageur's, and the price setter's optimization problems, which are solved simultaneously.

For each scenario $\omega$, the prosumer $n$ has to ensure the satisfaction of her individual power balance, enforced by (2b). Her demand $D_{n}$ has to be met by the power exchange $p_{n}$ in the forward market, $\tilde{p}_{n \omega}$ in the spot market, and her PV power generation $\tilde{S}_{n \omega}$, which is a scenario-dependent parameter. Finally, (2c) and (2d) restrict power exchanges within the energy community by parameters $\bar{P}_{n}$. We introduce theoretical bounds $\bar{P}_{n}$ on power exchanges to achieve a closed and compact decision set for all players [12]. This will be necessary later for proving the existence of the game solution [35]. However, we select sufficiently large values for $\bar{P}_{n}$, and check the equilibrium solution a posteriori to ensure $(2 \mathrm{c})$ and $(2 \mathrm{~d})$ are always inactive.

Furthermore, the spatial arbitrageur $\mathcal{P}^{a r}$ minimizes her expected cost $J^{a r}$ from trading energy between the energy community and a retailer for energy as

$$
\begin{aligned}
& \operatorname{Min}_{p^{\mathrm{i}}, p^{\mathrm{e}}, \tilde{p}_{\omega}^{\mathrm{i}}, \tilde{p}_{\omega}^{\mathrm{e}}} J^{a r}=\underbrace{\left(C^{\mathrm{i}}-\lambda\right) p^{\mathrm{i}}}_{\text {Forward market import cost }}-\underbrace{\left(C^{\mathrm{e}}-\lambda\right) p^{\mathrm{e}}}_{\text {Forward market export cost }} \\
& +\sum_{\omega \in \Omega} \pi_{\omega}^{\Theta}[\underbrace{\left(\tilde{C}^{\mathrm{i}}-\tilde{\lambda}_{\omega}\right) \tilde{p}_{\omega}^{\mathrm{i}}}_{\text {Spot market import cost }}-\underbrace{\left(\tilde{C}^{\mathrm{e}}-\tilde{\lambda}_{\omega}\right) \tilde{p}_{\omega}^{\mathrm{e}}}_{\text {Spot market export cost }}] \\
& \text { s.t. } 0 \leq p^{\mathrm{i}} \leq \bar{P}^{\mathrm{i}}: \underline{\chi}^{\mathrm{p}^{\mathrm{i}}}, \bar{\chi}^{\mathrm{p}^{\mathrm{i}}} \text {, } \\
& 0 \leq p^{\mathrm{e}} \leq \bar{P}^{\mathrm{e}}: \underline{\chi}^{\mathrm{p}^{\mathrm{e}}}, \bar{\chi}^{\mathrm{p}^{\mathrm{e}}}, \\
& 0 \leq \tilde{p}_{\omega}^{\mathrm{i}} \leq \bar{P}^{\mathrm{i}}: \underline{\chi}_{\omega}^{\tilde{p}^{\mathrm{i}}}, \bar{\chi}_{\omega}^{\tilde{\mathrm{p}}^{\mathrm{i}}}, \forall \omega, \\
& 0 \leq \tilde{p}_{\omega}^{\mathrm{e}} \leq \bar{P}^{\mathrm{e}}: \underline{\chi}_{\omega}^{\tilde{\mathrm{p}}^{\mathrm{e}}}, \bar{\chi}_{\omega}^{\tilde{\mathrm{p}}^{\mathrm{e}}}, \forall \omega .
\end{aligned}
$$

The first term of the objective function (3a) corresponds to the cost in the forward market from importing power $p^{\mathrm{i}}$ at the fixed price $C^{\mathrm{i}}$ while being paid at the local forward marketclearing price $\lambda$. Similarly, the second term represents the cost from exporting energy $p^{\mathrm{e}}$ at the forward market-clearing price $\lambda$, while receiving the fixed exporting price $C^{\mathrm{e}}$. The risk-

\footnotetext{
${ }^{6}$ Very small values for $\beta$ do not alter the total operational cost of the energy community in comparison to $\beta=0$. However, $\beta=0$ yields linear objective functions, and may give rise to multiple trading solutions for players [38]. We introduce regularizers to ensure strictly monotone objective functions. This allows us to draw conclusions on the uniqueness of the solution. In fact, a very small value for the regularizer ensures identical cost for players, who have identical risk preferences, production, and consumption profiles.
} 
neutral spatial arbitrageur weights local spot market scenarios according to physical probabilities $\pi_{\omega}^{\Theta}$. The third and fourth terms of (3a) resemble the cost from energy arbitrage between the energy community and the retailer under each scenario of the spot market, where $\tilde{C}^{\mathrm{i}}$ and $\tilde{C}^{\mathrm{e}}$ are fixed importing and exporting prices, respectively. Constraints (3b)-(3e) set bounds for importing $\bar{P}^{\mathrm{i}}$ and exporting $\bar{P}^{\mathrm{e}}$ power to and from the energy community.

Finally, for given values of $p_{n}, p^{\mathrm{i}}, p^{\mathrm{e}}, \tilde{p}_{n \omega}, \tilde{p}_{\omega}^{\mathrm{i}}$, and $\tilde{p}_{\omega}^{\mathrm{e}}$, the price-setter $\mathcal{P}^{p s}$ derives forward and spot market-clearing prices, i.e., $\lambda$ and $\tilde{\lambda}_{\omega}$, as

$$
\begin{aligned}
& \operatorname{Min}_{\lambda, \tilde{\lambda}_{\omega}} J^{p s}=\underbrace{\lambda\left(\sum_{n \in N} p_{n}-p^{\mathrm{i}}+p^{\mathrm{e}}\right)}_{\text {Buyers' forward market cost/ sellers' forward market revenue }} \\
& \quad+\sum_{\omega \in \Omega} \pi_{\omega}^{\Theta}\left[\tilde{\lambda}_{\omega}\left(\sum_{n \in N} \tilde{p}_{n \omega}-\tilde{p}_{\omega}^{\mathrm{i}}+\tilde{p}_{\omega}^{\mathrm{e}}\right)\right] \\
& \text { s.t. }-\bar{\Lambda} \leq \lambda \leq \bar{\Lambda}: \underline{\chi}^{\lambda}, \bar{\chi}^{\lambda}, \\
& -\bar{\Lambda} \leq \tilde{\lambda}_{\omega} \leq \bar{\Lambda}: \underline{\chi}_{\omega}^{\tilde{\lambda}}, \bar{\chi}_{\omega}^{\tilde{\lambda}}, \forall \omega .
\end{aligned}
$$

The first line of the objective function (4a) minimizes the cost for energy buyers and maximizes the revenue for energy sellers in the local forward market. By sellers, we refer to producers and energy imports. Similarly, by buyers, we refer to consumers and energy exports. The second line minimizes/maximizes the expected cost/revenue in the local spot market, where each scenario is weighted by the physical probability $\pi_{\omega}^{\Theta}$. Constraints (4b) and (4c) set the lower and upper bounds on market-clearing prices ${ }^{7}$. Note that in the case that (4b) and (4c) are inactive, the KKT conditions of (4) enforce power balance conditions within the energy community in both forward and spot markets.

Definition 1. Given optimization problems (2)-(4), we define $\Gamma\left(\mathcal{Z}, K,\left\{J_{i}\right\}_{\forall i \in \mathcal{Z}}\right)$ as the risk-neutral non-cooperative Nash game, where $\mathcal{Z}=\left(\mathcal{P} \cup \mathcal{P}^{a r} \cup \mathcal{P}^{p s}\right)$ is the set of all players. $K=\left(K_{1} \times \cdots \times K_{\mathcal{P}} \times K^{a r} \times K^{p s}\right)$ denotes the strategy set of the game, where $K_{i}$ is the strategy set of player $i \in \mathcal{Z}$.

Proposition 1. For the risk-neutral non-cooperative Nash game $\Gamma(\cdot)$ an equivalent optimization problem exists. In addition, the Nash equilibrium solution is unique.

Proof 1. We provide the proof in Appendix A-A.

\section{Market Clearing With Risk-Averse Players AND RISK TRADING}

We extend optimization problems of risk-neutral prosumers $n \in \mathcal{P}$ and the risk-neutral spatial arbitrageur $\mathcal{P}^{\text {ar }}$ by adding the coherent risk measure function $\rho(\cdot)$ as stated in (1a) over local spot market scenarios. The risk set of each player $\mathcal{D}^{\mathrm{CVaR}}$ is built upon the $\mathrm{CVaR}$ measure as defined

\footnotetext{
${ }^{7}$ Again, we theoretically consider a price floor and a price cap to achieve a compact and closed decision set for the price-setter, and thereby, to mathematically prove the solution existence. In our numerical study, we will consider a very large value for parameter $\bar{\Lambda}$ to ensure bounds are inactive. We refer the interested reader to [39], addressing how an active price cap may cause market inefficiency.
}

\begin{tabular}{llll} 
Risk-neutral Nash game $\Gamma(\cdot)$ & Risk-averse Nash game $\Gamma^{\rho}(\cdot)$ \\
\hdashline Prosumers $n \in \mathcal{P}:$ & $(2)$ & Risk aversion & Prosumers $n \in \mathcal{P}^{\rho}:$ \\
\hdashline Spatial arbitrageur $\mathcal{P}^{a r}:(3)$ & and & Spatial arbitrageur $\mathcal{P}^{a r, \rho}:(7),(8)$ \\
\hdashline Price-setter $\mathcal{P}^{p s}:$ & (4) & risk trading & Price-setter $\mathcal{P}^{p s, \cap:}$
\end{tabular}

Fig. 2: By adding risk aversion and risk trading to the risk-neutral Nash game, each player simultaneously solves two optimization problems within the riskaverse Nash equilibrium problem with risk trading.

in (1b). Moreover, we introduce risk trading among prosumers and the spatial arbitrageur. Finally, we extend the optimization problem of the price-setter $\mathcal{P}^{p s}$ such that she clears the market and respects prosumers' and the spatial arbitrageur's risk preferences when revealing local market-clearing prices. Figure 2 depicts the affiliation of optimization problems moving from the risk-neutral Nash game $\Gamma(\cdot)$ with one optimization problem per player to the risk-averse Nash game $\Gamma^{\rho}(\cdot)$ with risk trading, where we consider two optimization problems per player.

We first start with risk-averse prosumers $n \in \mathcal{P}^{\rho}$. Each prosumer is able to hedge the risk induced by the uncertain PV power generation in scenario $\omega$ by trading Arrow-Debreu securities $a_{n \omega}$ in the local forward market. We develop two optimization problems related to each risk-averse prosumer. These two problems are solved simultaneously within the Nash equilibrium problem. The first problem of prosumer $n$ minimizes her risk-adjusted expected cost $J_{n}^{\rho_{1}}$ by determining her trading decisions $p_{n}, \tilde{p}_{n \omega}$, and $a_{n \omega}$ while risk-adjusted probabilities $\pi_{n \omega}^{\rho}$ are given. The second one exhibits the dual representation (1a) of the $\mathrm{CVaR}$ as risk measure function $\rho^{\mathrm{CVaR}}(\cdot)$ and determines risk-adjusted probabilities, while her trading decisions are treated as fixed parameters. The first problem writes as

$$
\begin{gathered}
\{\underbrace{\operatorname{Min}}_{p_{n}, \tilde{p}_{n \omega}, a_{n \omega}} J_{n}^{\rho_{1}}=\lambda p_{n}+\frac{1}{2} \beta p_{n}^{2}+\underbrace{\sum_{\omega \in \Omega} \mu_{\omega} a_{n \omega}}_{\text {Forward market hedging cost/revenue }} \\
\quad+\sum_{\omega \in \Omega} \pi_{n \omega}^{\rho}(\tilde{\lambda}_{\omega} \tilde{p}_{n \omega}+\frac{1}{2} \beta \tilde{p}_{n \omega}^{2}-\underbrace{a_{n \omega}}_{\text {Hedging cost/revenue in } \omega}) \quad(5 \mathrm{a}) \\
\text { s.t. (2b) }-(2 \mathrm{~d})\} \forall n .
\end{gathered}
$$

Compared to (2a) in the risk-neutral setting, the objective function (5a) includes risk-adjusted probabilities $\pi_{n \omega}^{\rho}$ instead of physical ones. It also comprises one additional free variable per scenario, i.e., Arrow-Debreu security $a_{n \omega}$, and two additional cost/revenue components related to such securities. The first line of (5a) includes $\sum_{\omega \in \Omega} \mu_{\omega} a_{n \omega}$, which refers to the total cost/revenue of prosumer $n$ over scenarios in the local forward market, obtained by trading securities $a_{n \omega}$ at the risk price $\mu_{\omega}$. A positive value for $a_{n \omega}$ implies that prosumer $n$ buys securities in the forward market for scenario $\omega$, and thereby lowers her associated risk under that scenario. In contrast, a negative value for $a_{n \omega}$ means that prosumer $n$ sells securities in the forward market, and therefore is willing to accept a higher risk under scenario $\omega$. Note that similar to $\lambda$ and $\tilde{\lambda}_{\omega}$, the risk price $\mu_{\omega}$ is a parameter in (5), while it is a variable within the Nash equilibrium problem. Note also that $a_{n \omega}$ and $\mu_{\omega}$ are Nash equilibrium variables in the forward 
stage, though they are indexed by scenario $\omega$. If scenario $\omega$ occurs in the local spot market, the seller/buyer of security $a_{n \omega}$ pays/is paid at price 1 , as given in the second line of (5a).

The second optimization problem corresponding to each risk-averse prosumer $n \in \mathcal{P}^{\rho}$ resembles the risk measure function $\rho^{\mathrm{CVaR}}(\cdot)$, takes her scenario-indexed trading decisions $\tilde{p}_{n \omega}$ and $a_{n \omega}$ into account as parameters, and endogenously determines her risk-adjusted probabilities $\pi_{n \omega}^{\rho}$ as

$$
\begin{aligned}
\left\{\operatorname{Min}_{n \omega}^{\rho}\right. & J_{n}^{\rho_{2}}=-\sum_{\omega \in \Omega} \pi_{n \omega}^{\rho}\left(\tilde{\lambda}_{\omega} \tilde{p}_{n \omega}+\frac{1}{2} \beta \tilde{p}_{n \omega}^{2}-a_{n \omega}\right) \\
\text { s.t. } & \sum_{\omega \in \Omega} \pi_{n \omega}^{\rho}-1=0: \phi_{n}^{\rho}, \\
& \left.0 \leq \pi_{n \omega}^{\rho} \leq \frac{1}{\alpha_{n}} \pi_{\omega}^{\Theta}: \underline{\chi}_{n \omega}^{\rho}, \bar{\chi}_{n \omega}^{\rho}, \forall \omega\right\} \forall n,
\end{aligned}
$$

where the objective function (6a) minimizes the negative expected cost in the local spot market by optimally choosing values for risk-adjusted probabilities $\pi_{n \omega}^{\rho}$. Note that according to the definition of a coherent risk measure in (1a), the objective function (6a) maximizes the disbenefit for prosumer $n$ in the local spot market by increasing the weight of the worst scenarios. Constraints (6b) and (6c) set the bounds of riskadjusted probabilities $\pi_{n \omega}^{\rho}$ according to the risk set $\mathcal{D}_{n}^{\mathrm{CVaR}}$ of each risk-averse prosumer $n$.

Similarly, as for risk-averse prosumers we consider two optimization problems for the risk-averse spatial arbitrageur $\mathcal{P}^{a r}$, who is also able to participate in risk trading. In fact, she minimizes her risk-adjusted expected cost $J^{a r, \rho_{1}}$ as

$$
\begin{aligned}
& \operatorname{Min}_{\rho^{\mathrm{i}}, p^{\mathrm{e}}, \tilde{p}_{\omega}^{\mathrm{i}}, \tilde{p}_{\omega}^{\mathrm{e}}, b_{\omega}} J^{a r, \rho_{1}}=\left(C^{\mathrm{i}}-\lambda\right) p^{\mathrm{i}}-\left(C^{\mathrm{e}}-\lambda\right) p^{\mathrm{e}}+\underbrace{\sum_{\omega \in \Omega} \mu_{\omega} b_{\omega}}_{\text {Forward market hedging cost/revenue }} \\
& +\sum_{\omega \in \Omega} \pi_{\omega}^{a r}[\left(\tilde{C}^{\mathrm{i}}-\tilde{\lambda}_{\omega}\right) \tilde{p}_{\omega}^{\mathrm{i}}-\left(\tilde{C}^{\mathrm{e}}-\tilde{\lambda}_{\omega}\right) \tilde{p}_{\omega}^{\mathrm{e}}-\underbrace{b_{\omega}}_{\text {Hedging cost/revenue in } \omega}] \\
& \text { s.t. (3b) - (3e). }
\end{aligned}
$$

Compared to (3a) in the risk-neutral model, the first line of (7a) includes the total cost/revenue for the spatial arbitrageur from trading Arrow-Debreu securities $b_{\omega}$ at the risk price $\mu_{\omega}$ in the local forward market. The second line refers to her expected cost in the local spot market weighted by riskadjusted probabilities $\pi_{\omega}^{a r}$, including the hedging cost/revenue under each scenario $\omega$.

Similar to (6), the second optimization problem of the spatial arbitrageur within the Nash equilibrium problem determines her risk-adjusted probabilities $\pi_{\omega}^{a r}$, while treating her scenario-indexed trading decisions $\tilde{p}_{\omega}^{\mathrm{i}}, \tilde{p}_{\omega}^{\mathrm{e}}$, and $b_{\omega}$ as parameters. This problem writes as

$$
\begin{aligned}
\operatorname{Min}_{\omega}^{a r} & J^{a r, \rho_{2}}=-\sum_{\omega \in \Omega} \pi_{\omega}^{a r}\left[\left(\tilde{C}^{\mathrm{i}}-\tilde{\lambda}_{\omega}\right) \tilde{p}_{\omega}^{\mathrm{i}}-\left(\tilde{C}^{\mathrm{e}}-\tilde{\lambda}_{\omega}\right) \tilde{p}_{\omega}^{\mathrm{e}}-b_{\omega}\right] \\
\text { s.t. } & \sum_{\omega \in \Omega} \pi_{\omega}^{a r}-1=0: \phi^{a r}, \\
& 0 \leq \pi_{\omega}^{a r} \leq \frac{1}{\alpha^{a r}} \pi_{\omega}^{\Theta}: \underline{\chi}_{\omega}^{a r}, \bar{\chi}_{\omega}^{a r}, \forall \omega .
\end{aligned}
$$

The objective function ( $8 \mathrm{a}$ ) minimizes the negative expected local spot market cost $J^{a r, \rho_{2}}$ by choosing $\pi_{\omega}^{a r}$. Constraints (8b) and (8c) ensure bounds for risk-adjusted probabilities $\pi_{\omega}^{a r}$ according to the risk set $\mathcal{D}^{\mathrm{CVaR}, a r}$ of the risk-averse spatial arbitrageur.

Finally, the price-setter $\mathcal{P}^{p s, \cap}$ also considers two optimization problems within the Nash equilibrium problem. In the first optimization problem, she minimizes the risk-adjusted expected cost for energy/security buyers, maximizes the riskadjusted expected revenue for energy/security sellers, and determines market-clearing prices $\lambda, \tilde{\lambda}_{\omega}$ and $\mu_{\omega}$ as

$$
\begin{gathered}
\operatorname{Min}_{\lambda, \tilde{\lambda}_{\omega}, \mu_{\omega}} J^{p s, \cap_{1}}=\lambda\left(\sum_{n \in N} p_{n}-p^{\mathrm{i}}+p^{\mathrm{e}}\right)+\sum_{\omega \in \Omega} \mu_{\omega}\left(\sum_{n \in N} a_{n \omega}+b_{\omega}\right) \\
\quad+\sum_{\omega \in \Omega} \pi_{\omega}^{\cap}\left[\tilde{\lambda}_{\omega}\left(\sum_{n \in N} \tilde{p}_{n \omega}-\tilde{p}_{\omega}^{\mathrm{i}}+\tilde{p}_{\omega}^{\mathrm{e}}\right)\right] \\
\text { s.t. (4b) }-(4 \mathrm{c}), \\
\quad-\bar{M} \leq \mu_{\omega} \leq \bar{M}: \underline{\chi}_{\omega}^{\mu}, \bar{\chi}_{\omega}^{\mu}, \forall \omega .
\end{gathered}
$$

For given values of Arrow-Debreu securities $a_{n \omega}$ traded by prosumers $n \in \mathcal{P}^{\rho}$ as well as securities $b_{\omega}$ traded by the spatial arbitrageur for scenarios $\omega \in \Omega$, the price-setter chooses risk prices $\mu_{\omega}$, such that the cost for buyers is minimized and the revenue for sellers is maximized. Moreover, she weights cost in the local spot market according to system-wide risk-adjusted probabilities $\pi_{\omega}^{\bigcap}$, which are considered as parameters in (9). Constraint (9c) imposes theoretical lower and upper bounds for risk prices $\mu_{\omega}$.

In the second optimization problem, the price-setter determines the system-wide risk-adjusted probabilities $\pi_{\omega}^{\cap}$ given scenario-indexed trading decisions $\tilde{p}_{n \omega}, \tilde{p}_{\omega}^{\mathrm{i}}$, and $\tilde{p}_{\omega}^{\mathrm{e}}$, as well as risk sets of prosumers $\mathcal{D}_{n}^{\mathrm{CVaR}}$ and the spatial arbitrageur $\mathcal{D}^{\mathrm{CVaR}, a r}$ as

$$
\begin{aligned}
& \operatorname{Min}_{\pi_{\omega}^{n}} J^{p s, \cap_{2}}=-\sum_{\omega \in \Omega} \pi_{\omega}^{\cap}\left[\tilde{\lambda}_{\omega}\left(\sum_{n \in N} \tilde{p}_{n \omega}-\tilde{p}_{\omega}^{\mathrm{i}}+\tilde{p}_{\omega}^{\mathrm{e}}\right)\right] \\
& \text { s.t. } \sum_{\omega \in \Omega} \pi_{\omega}^{\cap}-1=0: \phi^{\cap} \text {, } \\
& 0 \leq \pi_{\omega}^{\cap} \leq \frac{1}{\alpha^{\cap}} \pi_{\omega}^{\Theta}: \underline{\chi}_{\omega}^{\cap}, \bar{\chi}_{\omega}^{\cap}, \forall \omega .
\end{aligned}
$$

Aligned with the definition of a coherent risk measure in (1a), the objective function (10a) maximizes disbenefits. In other words, it minimizes the expected energy revenue of sellers and maximizes the energy cost of buyers in the local spot market, aiming at determining system-wide riskadjusted probabilities $\pi_{\omega}^{\bigcap}$. Constraints (10b) and (10c) ensure bounds for system-wide risk-adjusted probabilities $\pi_{\omega}^{\bigcap}$, given the risk set $\mathcal{D}^{\mathrm{CVaR}, \cap}$. The risk set $\mathcal{D}^{\mathrm{CVaR}, \cap}$ is formed based on the intersection of risk sets of prosumers and the spatial arbitrageur. In fact, this risk set corresponds to the risk set of the least risk-averse player as described in [14]. This implies that $\alpha^{\cap}$ in (10c) is equal to $\min \left\{\alpha_{1}, \cdots, \alpha_{N}, \alpha^{a r}\right\}$.

Definition 2. Given (5)-(10), we define $\Gamma^{\rho}\left(\mathcal{Z}, K,\left\{J_{i}\right\}_{\forall i \in \mathcal{Z}}\right)$ as the risk-averse non-cooperative Nash game, where $\mathcal{Z}=$ $\left(\mathcal{P}^{\rho} \cup \mathcal{P}^{a r, \rho} \cup \mathcal{P}^{p s, \cap}\right)$ is the set of all players. $K=\left(K_{1} \times \cdots \times\right.$ $\left.K_{\mathcal{P}^{\rho_{1,2}}} \times K^{a r, \rho_{1,2}} \times K^{p s, \cap_{1,2}}\right)$ denotes the strategy set of the game, where $K_{i}$ is the strategy set of player $i \in \mathcal{Z}$.

Remark 2. The risk-averse Nash game $\Gamma^{\rho}(\cdot)$ with risk trading provides a generalized representation. By adjusting the risk aversion parameter of players, i.e., $\alpha_{1}, \cdots, \alpha_{\mathcal{P}^{\rho}}, \alpha^{a r}$, and 
TABLE II: A summary of problems to be solved.

\begin{tabular}{ll}
\hline & Problem \\
\hline Fully incomplete & Mixed complementarity problem (15)-(20) \\
Partially incomplete & Mixed complementarity problem (15)-(20) \\
Complete & Optimization (21), followed by optimization (22) \\
Neutral & Optimization (12) \\
\hline
\end{tabular}

the availability of Arrow-Debreu securities, $\Gamma^{\rho}(\cdot)$ is able to describe several game setups, including both risk-neutral and risk-averse Nash games. The risk-averse setups range from a fully incomplete market for risk, where no Arrow-Debreu security is available for trading, to a complete market for risk, where Arrow-Debreu securities are available for trading in all scenarios. The intermediate setups represent partially incomplete markets for risk, where Arrow-Debreu securities are only available for trading in a subset of scenarios.

Proposition 2. If the risk-averse non-cooperative Nash game $\Gamma^{\rho}(\cdot)$ is either fully or partially incomplete for risk, then no equivalent optimization problem necessarily exists. In addition, multiple Nash equilibria may exist.

Proof 2. We provide the proof in Appendix A-B.

Proposition 3. If the risk-averse non-cooperative Nash game $\Gamma^{\rho}(\cdot)$ is complete for risk, then an equivalent optimization problem exists, where all players reach a consensus on risk-adjusted probabilities due to unconstrained risk trading. This is in line with the findings of [14], [15], and [18]. However, multiple Nash equilibria may still exist, since objective functions are not strongly convex in $a_{n \omega}$ and $b_{\omega}$.

Proof 3. We provide the proof in Appendix A-C.

Remark 3. We reformulate the risk-averse Nash game $\Gamma^{\rho}(\cdot)$ with risk trading as a mixed complementarity problem [9] by concatenating KKT conditions from all optimization problems within $\Gamma^{\rho}(\cdot)$, i.e., optimization problems (5)-(10). Appendix B provides the full formulation of the resulting mixed complementarity problem. This mixed complementarity problem contains bilinear terms due to products of risk-adjusted probabilities and trading decisions, resulting in a mixed non-linear complementarity problem. However, it can be solved using PATH [40] or other mixed complementarity problem solvers.

Remark 4. The risk-averse Nash game $\Gamma^{\rho}(\cdot)$ with a complete market for risk can be solved in the same way as stated in Remark 3. However, Proposition 3 shows that an equivalent optimization problem for such a problem exists, which is a risk-averse social planner problem endowed with the risk measure function $\rho^{\bigcap}(\cdot)$ and the risk set $\mathcal{D}^{\mathrm{CVaR}, \cap}$. We provide such an optimization problem related to our risk-averse Nash game $\Gamma^{\rho}(\cdot)$ with a complete market for risk in Appendix C. By solving this convex optimization we avoid computational issues, coming along with solvers for mixed complementarity problems as highlighted in [17].

According to Remark 3 and Remark 4, we solve optimization problems for the risk-neutral Nash game $\Gamma(\cdot)$ and for the risk-averse Nash game $\Gamma^{\rho}(\cdot)$ when the market is complete for risk. In contrast, we solve mixed complementarity problems
TABLE III: Average computational time in seconds.

\begin{tabular}{rrrrr}
\hline & Fully incomp. & Partially incomp. & Complete & Neutral \\
\hline 6 Scenarios & 2.1 & 1.8 & 0.4 & 0.2 \\
500 Scenarios & 437.1 & 891.0 & 5.1 & 4.0 \\
\hline
\end{tabular}

associated with $\Gamma^{\rho}(\cdot)$ when the market is partially or fully incomplete for risk. We provide a summary of problems to be solved in Table II.

\section{Numerical Study AND Discussion}

We apply models presented in Sections III and IV to analyze the impact of heterogeneous risk aversion within an energy community. We start with an illustrative example with 6 scenarios to gain insights into risk trading. In the second part, we turn our attention to a problem with 500 scenarios, where we shed light on the payment flows among energy community members in the local forward and spot markets. Moreover, we gradually increase the risk aversion of one prosumer to highlight the implication for rival players.

Throughout this section, we consider three players, namely $n_{1}, n_{2}$, and $n_{3}$, in addition to the spatial arbitrageur $a r$. Player $n_{1}$ is a prosumer who owns a stochastic PV system with a mean power generation of $6 \mathrm{~kW}$ while having a deterministic demand of $10 \mathrm{~kW}$. Player $n_{2}$ owns a stochastic PV system only, with a mean power generation of $6 \mathrm{~kW}$. Finally, player $n_{3}$ is an inelastic demand, with a deterministic load of $10 \mathrm{~kW}$.

We consider the spatial arbitrageur to be the least risk-averse player with $\alpha^{a r}=0.9$. Player $n_{1}$ is moderately risk-averse with $\alpha_{1}=0.5$. Player $n_{2}$ is highly risk-averse with $\alpha_{2}=0.3$, while player $n_{3}$ is slightly risk-averse with $\alpha_{3}=0.7$. The fixed importing price $C^{\mathrm{i}}$ in the local forward market for the spatial arbitrageur is $€ 0.5 / \mathrm{kW}$, while she receives a price $C^{\mathrm{e}}$ of $€ 0.25 / \mathrm{kW}$ for exporting electricity in the forward market. In the local spot market, the fixed price for importing $\tilde{C}^{\mathrm{i}}$ is $€ 0.75 / \mathrm{kW}$, while the exporting price $\tilde{C}^{\mathrm{e}}$ is $€ 0.125 / \mathrm{kW}$. This incentivizes the energy community to optimally settle in the forward market, since costs are lower and revenues are higher. The regularizer is set to $\beta=0.01$. The lower and upper bounds for power trades and clearing prices-if unequal to zero-are chosen such that those bounds are never binding ${ }^{8}$. Lastly, for a partially incomplete market for risk, Arrow-Debreu securities are available for a third of the scenarios only.

\section{A. Computational Issues}

We use Gurobi Optimizer 9.0.1 under Python 3.7.6 to solve optimization problems, and PATH [40] under GAMS 24.6 to solve mixed complementarity problems. All these problems

\footnotetext{
${ }^{8}$ Note that upper bounds on imports and exports for the spatial arbitrageur represent the network capacity constraint between the energy community and the distribution system. These bounds, if active, alter local market-clearing prices. In particular, if upper bounds for energy imports and exports are binding and the energy community exhibits an energy surplus, local marketclearing prices decrease, while in the case of an energy shortage local market-clearing prices increase. Therefore, the network congestion changes the cost/revenue of players $n_{1}$ to $n_{3}$, while the spatial arbitrageur earns a profit from energy arbitrage. This profit comes from the price difference between the local market-clearing price and the buying/selling price of the retailer.
} 


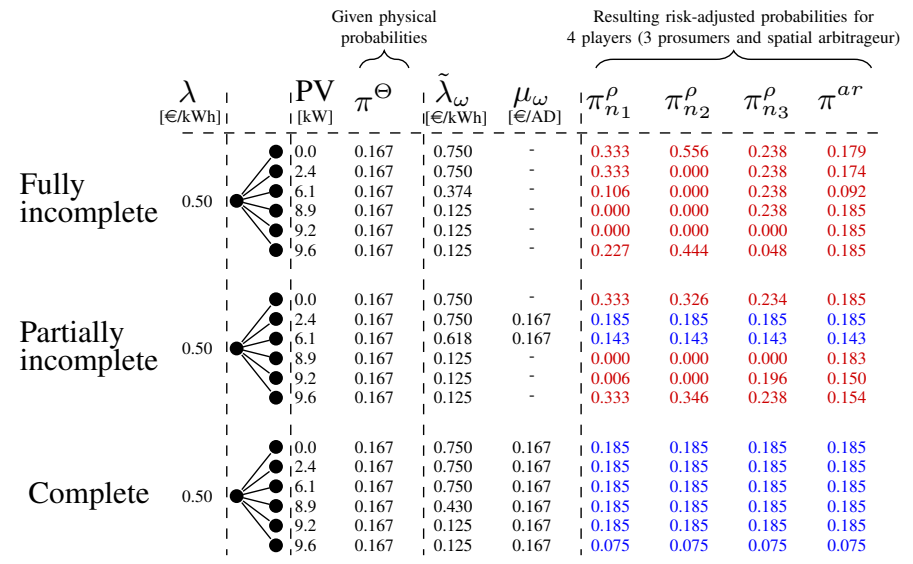

Fig. 3: Forward $\lambda$, spot $\tilde{\lambda}_{\omega}$, and Arrow-Debreu (AD) security $\mu_{\omega}$ marketclearing prices as well as risk-adjusted probabilities per player resulting from the stochastic PV power generation, which is reported per player, under different degrees of market completeness for risk, i.e., fully incomplete, partially incomplete, and complete. Equally weighted scenarios among four players are indicated in blue, while differently weighted scenarios are indicated in red.

are solved on a 8 GB-RAM computer clocking at $2.40 \mathrm{GHz}$. Note that all source codes are available in our online companion [41].

Optimization problems in general and mixed complementarity problems with at least one risk-neutral player scale well as the number of scenarios increases. Table III provides the computational time required to solve the underlying problem. For the case of 6 scenarios the computational time remains within seconds, while for the case of 500 scenarios the computational time needed by the PATH solver significantly increases. We observe computational challenges in the PATH solver when the problem size increases with respect to the number of scenarios. For such a case, an alternative solution algorithm is to use a decomposition approach, e.g., an alternating direction method of multipliers, to compute a risk-averse Nash equilibrium [42], though we leave it for future research.

We emphasize that the proposed Nash equilibrium problem does not serve as a tool for clearing a local energy market, where computational time significantly matters, but rather provides a framework for analyzing the implications of heterogeneous risk aversion and risk trading within local energy communities.

\section{B. Illustrative Example with 6 Scenarios}

Figure 3 presents the PV power generation $\tilde{S}_{n \omega}$ as scenariodependent input data, as well as optimization/Nash equilibrium problem outcomes for different degrees of market completeness for risk, including forward $\lambda$ and spot $\tilde{\lambda}_{\omega}$ market-clearing prices, risk prices $\mu_{\omega}$, and risk-adjusted probabilities of different players, i.e., $\pi_{n \omega}^{\rho}$ and $\pi_{\omega}^{a r}$. In the fully incomplete case, risk-adjusted probabilities vary highly among players. The most risk-averse player $n_{2}$ weights the scenario with zero PV power generation the most with $\pi_{n_{2} \omega_{1}}^{\rho}=0.556$, since no revenues are achieved. Interestingly, the weight of the scenario with the highest PV power generation is increased since this event causes the local spot market-clearing price to reduce, which also induces lower revenues. A similar behavior, but

TABLE IV: Power schedules in the local forward market [kW].

\begin{tabular}{lcccc}
\hline & $n_{1}$ & $n_{2}$ & $n_{3}$ & Spatial arbitrageur \\
\hline Fully incomplete & 3.72 & -2.27 & 5.34 & 6.79 \\
Partially incomplete & 2.91 & -2.15 & 4.89 & 5.65 \\
Complete & 2.00 & -2.99 & 4.86 & 3.87 \\
Neutral & 1.67 & -3.33 & 4.68 & 3.03 \\
\hline
\end{tabular}
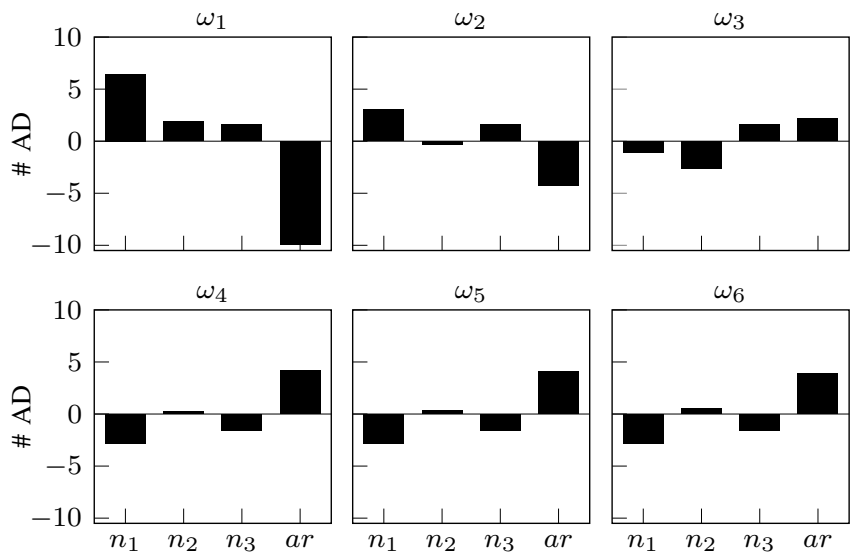

Fig. 4: Arrow-Debreu (AD) security trades among the three players $n_{1}$ to $n_{3}$ and the spatial arbitrageur $(a r)$ for all scenarios $\omega_{1}$ to $\omega_{6}$ in the forward stage of a complete local energy market for risk.

less extreme, is observed for player $n_{1}$. In contrast, player $n_{3}$, who is a sole consumer, increases weights for scenarios with high spot market-clearing prices while accordingly reducing weights of scenarios with low spot market-clearing prices. As we move towards the complete case, for scenarios where Arrow-Debreu securities are available, players reach a consensus on risk-adjusted probabilities, marked in the partially incomplete and complete cases in blue.

Based on risk-adjusted probabilities given in Figure 3, players adjust their forward market trades, as listed in Table IV. If the market is fully incomplete for risk, player $n_{2}$, who owns a stochastic PV system, reduces her local forward market trades and postpones trading decisions to be made in the local spot market when the realization of PV power generation is observed. In contrast, player $n_{3}$, who has a deterministic load, prefers meeting the majority of her demand in the local forward market, and thereby avoids the price volatility over local spot market scenarios. Player $n_{1}$ has a stochastic PV power generation as well as a deterministic load. We observe that she meets her demand in the local forward market while postponing decisions regarding $\mathrm{PV}$ power generation to the local spot market. The spatial arbitrageur imports/exports to/from the energy community according to trading strategies of players $n_{1}$ to $n_{3}$.

Risk trading outweighs heterogeneous risk aversion and shifts risk-adjusted forward trades towards observations in a risk-neutral setting. Figure 4 illustrates the Arrow-Debreu security trades in the forward stage for each scenario in a complete market for risk, which allow reaching a consensus on risk preferences. All three players $n_{1}$ to $n_{3}$ buy ArrowDebreu securities for scenario $\omega_{1}$ with the lowest PV power generation, while the least risk-averse player, who in our case is the spatial arbitrageur, is the only Arrow-Debreu security 


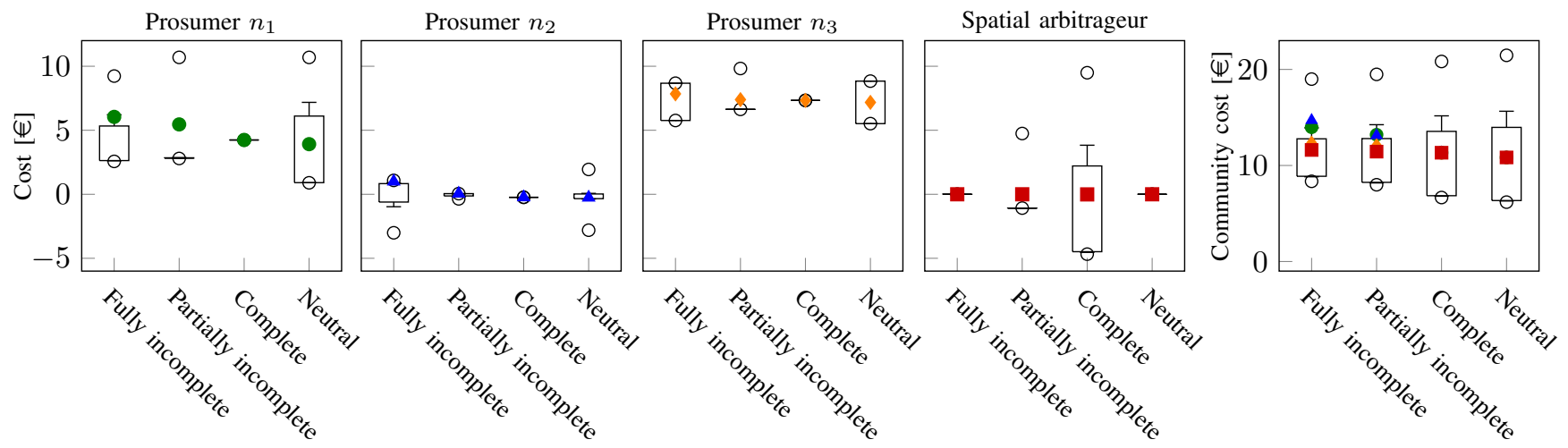

Fig. 5: Cost distribution over scenarios $\omega_{1}$ to $\omega_{6}$ for players as well as for the community as a whole in dependence of the degree of market completeness for risk. Colored markers refer to the risk-adjusted expected cost from each player's perspective. The distribution of the cost over scenarios is given by boxes, horizontal lines, and circles. Each box highlights the cost between the second and the third quartile. Horizontal lines show the 5th and 95th quantiles. Finally, circles indicate outliers below the 5th and beyond the 95th quantiles.

seller. However, for the following scenarios, we observe the opposite trend. In most cases, risk-averse players $n_{1}$ to $n_{3}$ emerge as Arrow-Debreu security sellers, while the spatial arbitrageur appears as a Arrow-Debreu security buyer. Therefore, players $n_{1}$ to $n_{3}$ erase their cost volatility, while the spatial arbitrageur absorbs it. Thus, unconstrained risk trading leads to a consensus on risk-adjusted probabilities corresponding to the risk preference of the least risk-averse player, i.e., the spatial arbitrageur.

The effect of risk trading on the cost distribution as well as the distribution of the total community cost is shown in Figure 5. Moving from a fully incomplete market to a complete market for risk, the cost distribution for players reduces to the point where in the complete case costs in all scenarios are identical. However, since the spatial arbitrageur absorbs the cost volatility of players $n_{1}$ to $n_{3}$, her cost volatility increases. Nevertheless, her expected cost remains unchanged. If all players are risk-neutral, they realize the lowest disbenefit. Similarly, looking at the total community cost we observe that moving from a fully incomplete market to a complete market for risk, the total expected community cost reduces by $5 \%$, while the community cost volatility increases. This cost reduction as a result of completing the market for risk confirms findings by [15] as discussed in Section II-D. Moreover, owning to risk trading, the expected community cost from each player's perspective converges towards a common belief, indicating a consensus on risk preferences.

\section{Assessing Risk Aversion with 500 Scenarios}

In this section we assume the spatial arbitrageur to be risk-neutral, i.e., $\alpha^{a r}=1$, because we expect the spatial arbitrageur to be a necessary automated service for the energy community in a similar way as the price setter clears the local market. Moreover, this assumption allows us to solve mixed complementarity problems with the PATH solver under GAMS without any computational issues as the number of scenarios increases.

Figure 6 illustrates the evolution of the expected community cost and its standard deviation in the risk-neutral Nash game

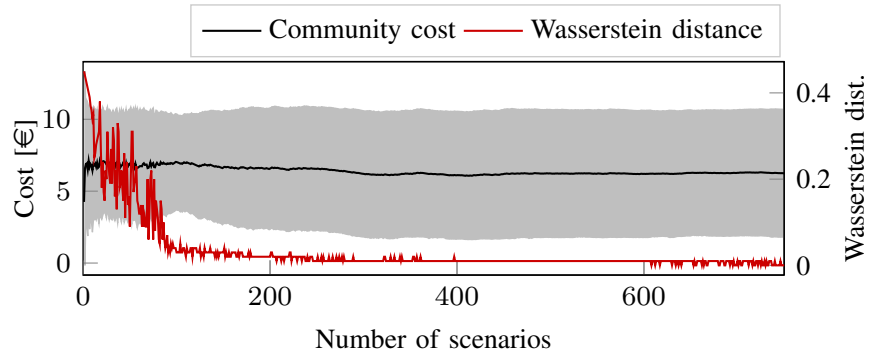

Fig. 6: Expected community cost and standard deviation highlighted by the shaded area in the risk-neutral Nash game. The Wasserstein distance shows the convergence against a good representation of the probability distribution as the number of scenarios increases.

with the number of scenarios. Moreover, we show the Wasserstein distance [43] between each set of sampled scenarios and its predecessor. Based on these numerical findings, we observe that from 500 scenarios onward changes arising from one additional scenario representing uncertain PV power generation hardly take place. Therefore, we choose 500 discrete scenarios to approximate the underlying continuous probability distribution of PV power generation.

Figure 7 presents the expected cost of all players as well as the total community cost. It provides details of costs specifically incurred in the local forward and spot markets by trading energy and Arrow-Debreu securities. Figure 7a corresponds to a fully incomplete market for risk, where players $n_{1}$ to $n_{3}$ incur the highest cost among all cases. This causes the expected community cost with $8.39 €$ to be comparatively high too.

As risk trading is possible for one-third of 500 scenarios, Figure $7 \mathrm{~b}$ illustrates how players $n_{1}$ to $n_{3}$ reduce their energy trades in the local forward market and increase more profitable activities in the local spot market, though subject to the PV power generation uncertainty. This uncertainty is partially hedged by awarded Arrow-Debreu securities. Note that the cost for all players $n_{1}$ to $n_{3}$ as well as the community cost decrease to some extent in comparison to those costs in the fully incomplete case. In particular, the expected community cost decreases from $8.39 €$ in the fully incomplete case to $7.62 €$ 


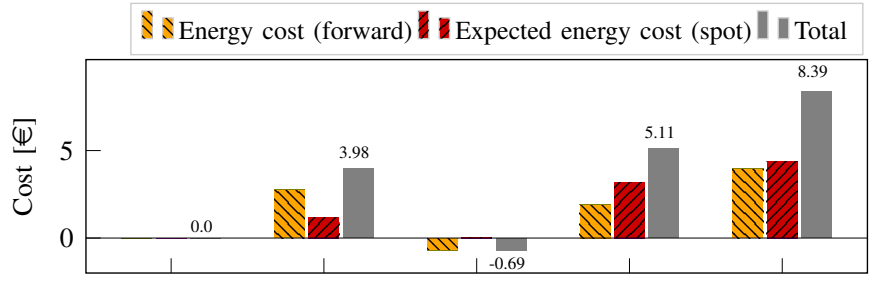

(a) Fully incomplete market for risk.

I| $\mathrm{AD}$ trading cost (forward) II Expected AD trading cost (spot)

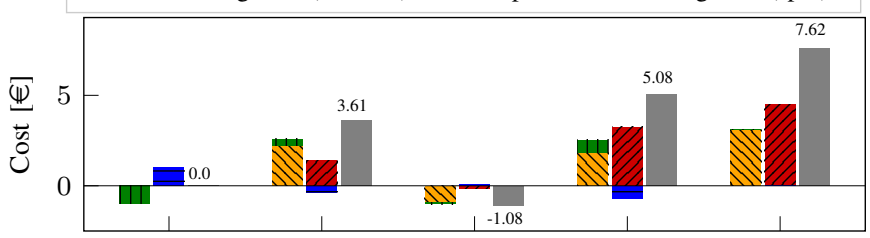

(b) Partially incomplete market for risk.

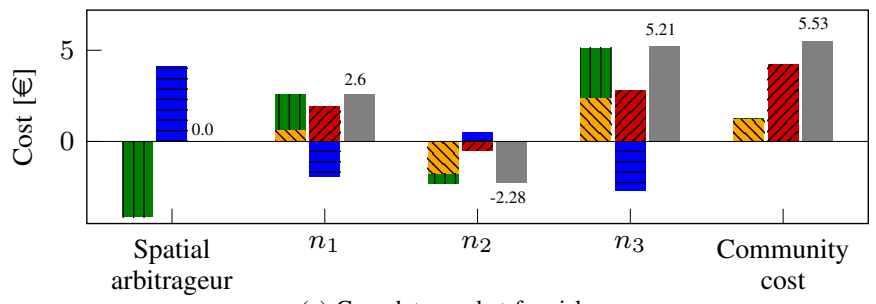

(c) Complete market for risk

Fig. 7: Expected cost of players $n_{1}, n_{2}, n_{3}$ and the spatial arbitrageur over 500 scenarios incurred by trading energy and Arrow-Debreu (AD) securities in local forward and spot markets. Plot 7 a corresponds to a fully incomplete market for risk, plot $7 \mathrm{~b}$ associates with a partially incomplete market for risk, and lastly, plot $7 \mathrm{c}$ refers to a complete market for risk.

in the partially incomplete case, implying a $9.18 \%$ saving in the community cost.

Figure $7 \mathrm{c}$ shows results for a case wherein the local energy market is complete for risk, i.e., risk trading is possible for all 500 scenarios. The three players $n_{1}$ to $n_{3}$ as well as the community as a whole incur the lowest cost among all three cases. In particular, the expected community cost drops to $5.53 €$, i.e., a $34.09 \%$ saving in the community cost compared to a fully incomplete market for risk. Moreover, this plot shows that energy trades in the local forward market have been reduced even further, while the engagement of players $n_{1}$ to $n_{3}$ in the local spot market has been increased. It is worth noting that the expected cost of each player in the local spot market is fully compensated by Arrow-Debreu securities. This causes a significant cost volatility for the spatial arbitrager, who is the greatest security seller in this case study.

Finally, we note that the spatial arbitrageur yields a zero cost in expectation in all three cases. In particular, since constraints on energy imports and exports are never binding, the local forward and spot market-clearing prices are equal to importing and exporting prices. Moreover, the revenue of the spatial arbitrageur from selling Arrow-Debreu securities in the local forward market are fully balanced with her expected cost in the local spot market for compensating the security buyers.

In the following, we gradually increase the risk aversion of one of the players, e.g., player $n_{1}$, and investigate impacts on her rivals. Figure $8 \mathrm{a}$ shows such an effect on the expected cost as well as the cost standard deviation. For the fully

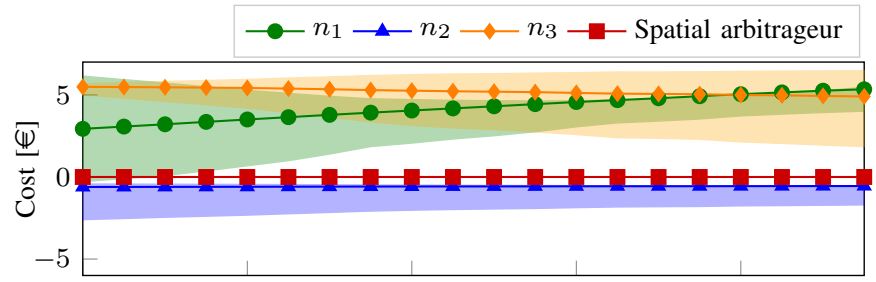

(a) Fully incomplete market for risk.

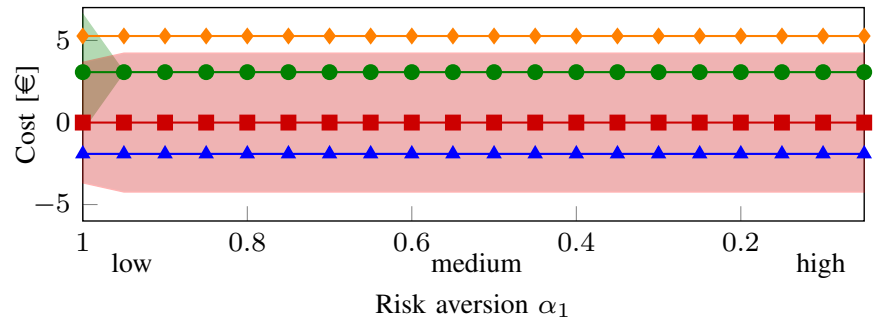

(b) Complete market for risk.

Fig. 8: Cost under an increasing risk aversion of player $n_{1}$. Plot 8 a graphs observations for a fully incomplete market for risk, while plot $8 \mathrm{~b}$ illustrates the situation for a complete market for risk. Marked lines refer to the expected cost while shaded areas highlight the cost standard deviation.

incomplete case the expected cost for player $n_{1}$ increases significantly as her risk aversion $\alpha_{1}$ increases. However, owning to her risk-adjusted forward market position the cost standard deviation decreases. Moreover, we observe that the risk aversion of player $n_{1}$ impacts the expected cost and the cost standard deviation of players $n_{2}$ and $n_{3}$. Player $n_{3}$ incurs comparatively lower expected cost, while her cost standard deviation increases. The reason for this is that player $n_{1}$ trades her stochastic PV power generation in the local spot market, and thereby lowers the local spot market-clearing price from which player $n_{3}$ profits.

As noted previously, if the market is complete for risk, riskaverse players $n_{1}$ to $n_{3}$ fully erase their cost volatility, while the spatial arbitrageur absorbs all the cost volatility as graphed in Figure 8b. Thus, the standard deviation of players $n_{1}$ to $n_{3}$ is zero, while the spatial arbitrageur experiences a remarkable cost volatility, though with an expected value of zero.

\section{CONClusion And Future Work}

This work proposes risk trading within energy communities to outweigh market inefficiencies arising from heterogeneously risk-averse community members. We have formulated a twostage stochastic Nash equilibrium problem and show different solution approaches depending on the degree of market completeness for risk. Risk trading complements local energy markets within energy communities when players have heterogeneous risk preferences. Risk trading protects players with slight risk aversion from conservative decisions made by highly risk-averse players. As a result, a significant system cost saving can be realized, while disbenefits for all players are reduce.

This work opens a wide range of research questions to be addressed in the future. The role of an energy storage system should be considered, since energy arbitrage over time can reduce the volatility of local market-clearing prices, and therefore the cost volatility for players by a different mean 
than risk trading. In addition, the small number of energy community members gives rise to potential strategic behavior by some local market participants. This aspect should not be neglected in designing a suitable risk trading product. If the spatial size of an energy community increases, network constraints within the community and resulting power losses have to be respected too. In addition, it is of interest to include the possibility of peer-to-peer energy trading among prosumers within the energy community. Another interesting research direction is to explore risk trading among retailers/aggregators on a distribution system level, i.e., one level above energy communities. Furthermore, we note that Arrow-Debreu security is a highly stylized financial product, which can only be traded for predefined scenarios. Therefore, the probability distribution of an uncertain event must be certainly known to complete the market for risk. Research on a less stylized product is of interest for determining an optimal trade-off between market completeness for risk and applicability of a financial product. Lastly, from a computational perspective, research on different solution algorithms for Nash equilibrium problems, e.g., based on various decomposition algorithms, is promising.

\section{APPENDIX A}

PROOFS

Proofs are based on equivalent forms of Variational Inequality (VI) problems and strict monotonicity of players' preferences [35].

\section{A. Proof of Proposition 1}

We state the problem $\Gamma(\cdot)$ of finding a Nash equilibrium as a $\operatorname{VI}(F, K)$ with the game map $F(z)=$ $\left[\nabla_{1} J_{1}\left(z_{1}, z_{-1}\right), \cdots, \nabla_{a r} J^{a r}\left(z_{a r}, z_{-a r}\right), \nabla_{\lambda} J^{\lambda}\left(z_{\lambda}, z_{-\lambda}\right)\right]^{\top}$, where $z=\left[p_{1}, \tilde{p}_{1 \omega}, \cdots, p^{\mathrm{i}}, p^{\mathrm{e}}, \tilde{p}_{\omega}^{\mathrm{i}}, \tilde{p}_{\omega}^{\mathrm{e}}, \lambda, \tilde{\lambda}_{\omega}\right]$ denotes the strategy vector. For the game $\Gamma(\cdot)$ the strategy set $K_{i}$ is compact, convex, and non-empty. Moreover, the game map $F(z)$ is continuous, since all cost functions $J_{i \in \mathcal{Z}}$ are continuously differentiable. Therefore, a solution set $\operatorname{SOL}(K, F)$ exists.

To show the singleton nature of the solution set $\operatorname{SOL}(K, F)$ we derive the Jacobian matrix of $F(z)$ as

$$
\nabla z F(z)=\left(\begin{array}{ccccccccc}
\hline \beta & 0 & \cdots & 0 & 0 & 0 & 0 & 1 & 0 \\
0 & \pi_{\omega}^{\Theta} \beta & \cdots & 0 & 0 & 0 & 0 & 0 & \pi_{\omega}^{\Theta} \\
\hline \vdots & \ddots & \ddots & \vdots & \vdots & \vdots & \vdots & \vdots & \vdots \\
0 & 0 & \cdots & 0 & 0 & 0 & 0 & -1 & 0 \\
0 & 0 & \cdots & 0 & 0 & 0 & 0 & 1 & 0 \\
0 & 0 & \cdots & 0 & 0 & 0 & 0 & 0 & -\pi_{\omega}^{\Theta} \\
0 & 0 & \cdots & 0 & 0 & 0 & 0 & 0 & \pi_{\omega}^{\Theta} \\
\hline 1 & 0 & \cdots & -1 & 1 & 0 & 0 & 0 & 0 \\
0 & \pi_{\omega}^{\Theta} & \cdots & 0 & 0 & -\pi_{\omega}^{\Theta} & \pi_{\omega}^{\Theta} & 0 & 0
\end{array}\right) J_{n}
$$

The Jacobian matrix above is symmetric indicated by the blue diagonal entries, meaning that the corresponding game is integrable [9]. This implies that an equivalent optimization problem solving the $\mathrm{VI}(F, K)$ exists, whose objective function is given by

$$
\theta(z)=\int_{0}^{1} F\left(z^{0}+t\left(z-z^{0}\right)\right)^{\top}\left(z-z^{0}\right) d t=
$$

$$
\begin{aligned}
& C^{\mathrm{i}} p^{\mathrm{i}}-C^{\mathrm{e}} p^{\mathrm{e}}+\sum_{n \in N} \frac{1}{2} \beta p_{n}^{2} \\
& +\sum_{\omega \in \Omega} \pi_{\omega}^{\Theta}\left(\tilde{C}^{\mathrm{i}} \tilde{p}_{\omega}^{\mathrm{i}}-\tilde{C}^{\mathrm{e}} \tilde{p}_{\omega}^{\mathrm{e}}+\sum_{n \in N} \frac{1}{2} \beta \tilde{p}_{n \omega}^{2}\right),
\end{aligned}
$$

which motivates the optimization problem

$$
\begin{aligned}
& \text { Min. } \\
& p_{n}, p^{\mathrm{i}}, p^{\mathrm{e}}, \tilde{p}_{n \omega}, \tilde{p}_{\omega}^{\mathrm{i}}, \tilde{p}_{\omega}^{\mathrm{e}} \\
& \text { s.t. (2b), (2c), (3b), (3d). }
\end{aligned}
$$

The objective function of the resulting optimization problem is convex and quadratic. This confirms that a unique Nash equilibrium point for the risk-neutral Nash game $\Gamma(\cdot)$ exists.

\section{B. Proof of Proposition 2}

Following Appendix A-A we state $\Gamma^{\rho}(\cdot)$ as $\mathrm{VI}^{\rho}(F, K)$ with $F(z)=\left[\nabla_{1} J_{1}^{\rho_{1}}\left(z_{1}, z_{-1}\right), \nabla_{1} J_{1}^{\rho_{2}}\left(z_{1}, z_{-1}\right), \cdots, \nabla_{a r} J^{a r, \rho_{1}}\left(z_{a r}, z_{-a r}\right)\right.$, $\left.\nabla_{a r} J^{a r, \rho_{2}}\left(z_{a r}, z_{-a r}\right), \nabla_{p s} J^{p s, \cap_{1}}\left(z_{p s}, z_{-p s}\right), \nabla_{p s} J^{p s, \cap_{2}}\left(z_{p s}, z_{-p s}\right)\right]^{\top}$, where $z=\left[p_{1}, \tilde{p}_{1 \omega}, a_{1 \omega}, \pi_{1 \omega}^{\rho}, \cdots, p^{\mathrm{i}}, p^{\mathrm{e}}, \tilde{p}_{\omega}^{\mathrm{i}}, \tilde{p}_{\omega}^{\mathrm{e}}, b_{\omega}, \pi_{\omega}^{a r}, \mu_{\omega}, \lambda, \tilde{\lambda}_{\omega}\right.$, $\left.\pi_{\omega}^{\cap}\right]$ denotes the strategy vector of the game. Moreover, to apply tools from VI we assume constraints on Arrow-Debreu security trades, although they are never binding. We write the Jacobian matrix as

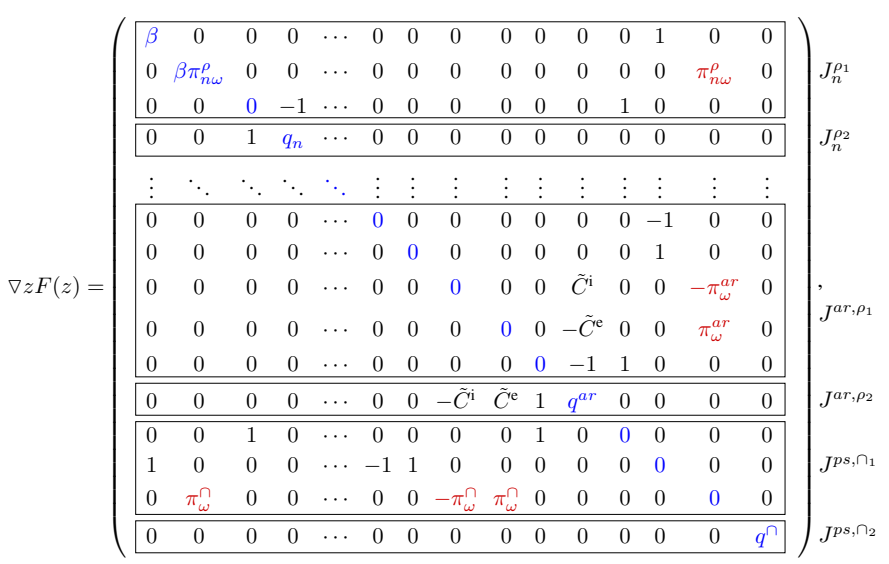

where $q_{n}=-\frac{1}{\pi_{n \omega}^{\rho}}\left(\tilde{\lambda}_{\omega} \tilde{p}_{n \omega}-\frac{1}{2} \beta \tilde{p}_{n \omega}^{2}\right), q^{a r}=\frac{1}{\pi_{\omega}^{a r}} \tilde{\lambda}_{\omega}\left(\tilde{p}_{\omega}^{\mathrm{i}}-\tilde{p}_{\omega}^{\mathrm{e}}\right)$, $q^{\cap}=-\frac{1}{\pi_{\omega}^{\cap}}\left(\frac{1}{2} \beta \tilde{p}_{n \omega}^{2}+\tilde{C}^{\mathrm{i}} \tilde{p}_{\omega}^{\mathrm{i}}-\tilde{C}^{\mathrm{e}} \tilde{p}_{\omega}^{\mathrm{e}}\right)$. Since the market is incomplete for risk, players potentially apply different riskadjusted probabilities. Therefore, the Jacobian matrix of $\Gamma^{\rho}(\cdot)$ is asymmetric as highlighted by the red entries. Thus, an equivalent optimization problem does not necessarily exist.

\section{Proof of Proposition 3}

If the market is complete for risk, all players apply identical risk-adjusted probabilities, i.e., $\pi_{n \omega}^{\rho}=\pi_{\omega}^{a r}=\pi_{\omega}^{\bigcap}$. This observation is based on the equivalence by the zero-gradient conditions with respect to Arrow-Debreu securities among all players for the case of unconstrained risk trading as given in Appendix B. Thus, the Jacobian matrix of $\Gamma^{\rho}(\cdot)$ is symmetric, with a skew symmetric inner part. An equivalent optimization problem solving $\mathrm{VI}^{\rho}(F, K)$ exists. Its objective is given by

$$
\theta(z)=\int_{0}^{1} F\left(z^{0}+t\left(z-z^{0}\right)\right)^{\top}\left(z-z^{0}\right) d t=
$$




$$
\begin{aligned}
& C^{\mathrm{i}} p^{\mathrm{i}}-C^{\mathrm{e}} p^{\mathrm{e}}+\sum_{n \in N} \frac{1}{2} \beta p_{n}^{2} \\
& +\sum_{\omega \in \Omega} \pi_{\omega}^{\cap}\left(\tilde{C}^{\mathrm{i}} \tilde{p}_{\omega}^{\mathrm{i}}-\tilde{C}^{\mathrm{e}} \tilde{p}_{\omega}^{\mathrm{e}}+\sum_{n \in N} \frac{1}{2} \beta \tilde{p}_{n \omega}^{2}\right),
\end{aligned}
$$

where $\pi_{\omega}^{\cap} \in \mathcal{D}^{\mathrm{CVaR}, \cap}$. This gives rise to

$$
\underset{p_{n}, p^{\mathrm{i}}, p^{\mathrm{e}}, \tilde{p}_{n \omega}, \tilde{p}_{\omega}^{\mathrm{i}}, \tilde{p}_{\omega}^{\mathrm{e}}}{\operatorname{Min}}(13)
$$

$$
\text { s.t. (2b), (2c), (3b), (3d). }
$$

However, we cannot derive a conclusion on the solution uniqueness, since Arrow-Debreu security trades are not explicitly stated in the optimization problem. Therefore, the objective function (13) is not strongly convex in $a_{n \omega}$ and $b_{\omega}$.

\section{APPENDIX B \\ MiXed Complementarity Problem}

We reformulate the Nash equilibrium problem as a mixed non-linear complementarity problem, which solves the riskaverse Nash game $\Gamma^{\rho}(\cdot)$ with risk trading. In the following, we provide this problem by concatenating the KKT conditions associated with prosumers' optimization problems (5)-(6), the spatial arbitrageur's optimization problems (7)-(8), and the price setter's optimization problems (9)-(10). Note that $\mathcal{L}$ refers to the Lagrangian function of the underlying optimization problem.

The KKT conditions associated with (5) are as follows. Note that in order to obtain a closed and compact decision set, we consider theoretical lower and upper bounds on Arrow-Debreu security trades in the form of $-\bar{Y}_{\omega} \leq a_{n \omega} \leq \bar{Y}_{\omega}, \forall n, \omega$, whose dual variables are $\underline{\chi}_{n \omega}^{\mathrm{a}}$ and $\bar{\chi}_{n \omega}^{\mathrm{a}}$, respectively.

$$
\begin{aligned}
& \frac{\partial \mathcal{L}}{\partial p_{n}}=\lambda+\beta p_{n}+\sum_{\omega \in \Omega} \tilde{\phi}_{n \omega}-\underline{\chi}_{n}^{\mathrm{p}}+\bar{\chi}_{n}^{\mathrm{p}}=0, \forall n \\
& \frac{\partial \mathcal{L}}{\partial \tilde{p}_{n \omega}}=\pi_{n \omega}^{\rho}\left(\tilde{\lambda}_{\omega}+\beta \tilde{p}_{n \omega}\right)+\tilde{\phi}_{n \omega}-\underline{\chi}_{n \omega}^{\tilde{\mathrm{p}}}+\bar{\chi}_{n \omega}^{\tilde{\tilde{p}}}=0, \forall n, \omega \\
& \frac{\partial \mathcal{L}}{\partial a_{n \omega}}=\mu_{\omega}-\pi_{n \omega}^{\rho}-\underline{\chi}_{n \omega}^{\mathrm{a}}+\bar{\chi}_{n \omega}^{\mathrm{a}}=0, \forall n, \omega \\
& 0 \leq p_{n}-\underline{P}_{n} \perp \underline{\chi}_{n}^{\mathrm{p}} \geq 0, \forall n \\
& 0 \leq \bar{P}_{n}-p_{n} \perp \bar{\chi}_{n}^{\mathrm{p}} \geq 0, \forall n \\
& 0 \leq \tilde{p}_{n \omega}-\underline{P}_{n} \perp \underline{\chi}_{n \omega}^{\tilde{\mathrm{p}}} \geq 0, \forall n, \omega \\
& 0 \leq \bar{P}_{n}-p_{n \omega}^{\tilde{\mathrm{p}}} \perp \bar{\chi}_{n \omega}^{\tilde{\tilde{p}}} \geq 0, \forall n, \omega \\
& 0 \leq a_{n \omega}+\bar{Y}_{\omega} \perp \underline{\chi}_{n \omega}^{\mathrm{a}} \geq 0, \forall n, \omega \\
& 0 \leq \bar{Y}_{\omega}-a_{n \omega} \perp \bar{\chi}_{n \omega}^{\mathrm{a}} \geq 0, \forall n, \omega \\
& p_{n}+\tilde{p}_{n \omega}+\tilde{S}_{n \omega}-D_{n}=0, \forall n, \omega
\end{aligned}
$$

The KKT conditions associated with (6) are

$$
\begin{aligned}
& \frac{\partial \mathcal{L}}{\partial \pi_{n \omega}^{\rho}}=-\left(\tilde{\lambda}_{\omega} \tilde{p}_{n \omega}+\frac{1}{2} \beta \tilde{p}_{n \omega}^{2}-a_{n \omega}\right)+\phi_{n}^{\rho}-\underline{\chi}_{n \omega}^{\rho}+\bar{\chi}_{n \omega}^{\rho} \\
& =0, \forall n, \omega, \\
& 0 \leq \pi_{n \omega}^{\rho} \perp \underline{\chi}_{n \omega}^{\rho} \geq 0, \forall n, \omega, \\
& 0 \leq \frac{1}{\alpha_{n}} \pi_{\omega}^{\Theta}-\pi_{n \omega} \perp \bar{\chi}_{n \omega}^{\rho} \geq 0, \forall n, \omega, \\
& \sum_{\omega \in \Omega} \pi_{n \omega}^{\rho}-1=0, \forall n \text {. }
\end{aligned}
$$

The KKT conditions corresponding to (7) are as follows. Again, in order to achieve a closed and compact decision set, we consider theoretical lower and upper bounds on ArrowDebreu security trades of the spatial arbitrageur in the form of $-\bar{Y}_{\omega} \leq b_{\omega} \leq \bar{Y}_{\omega}, \forall \omega$, whose dual variables are $\underline{\chi}_{\omega}^{\mathrm{b}}$ and $\bar{\chi}_{\omega}^{\mathrm{b}}$, respectively.

$$
\begin{aligned}
& \frac{\partial \mathcal{L}}{\partial p^{\mathrm{i}}}=C^{\mathrm{i}}-\lambda-\underline{\chi}^{\mathrm{p}^{\mathrm{i}}}+\bar{\chi}^{\mathrm{p}^{\mathrm{i}}}=0, \\
& \frac{\partial \mathcal{L}}{\partial p^{\mathrm{e}}}=-C^{\mathrm{e}}+\lambda-\underline{\chi}^{\mathrm{p}^{\mathrm{e}}}+\bar{\chi}^{\mathrm{p}^{\mathrm{e}}}=0, \\
& \frac{\partial \mathcal{L}}{\partial \tilde{p}_{\omega}^{\mathrm{i}}}=\pi_{\omega}^{a r}\left(\tilde{C}^{\mathrm{i}}-\tilde{\lambda}_{\omega}\right)-\underline{\chi}_{\omega}^{\tilde{\mathrm{p}}^{\mathrm{i}}}+\bar{\chi}_{\omega}^{\tilde{\tilde{p}}^{\mathrm{i}}}=0, \forall \omega, \\
& \frac{\partial \mathcal{L}}{\partial \tilde{p}_{\omega}^{\mathrm{e}}}=-\pi_{\omega}^{a r}\left(\tilde{C}^{\mathrm{e}}-\tilde{\lambda}_{\omega}\right)-\underline{\chi}_{\omega}^{\tilde{\mathrm{p}}^{\mathrm{e}}}+\bar{\chi}_{\omega}^{\tilde{\mathrm{p}}^{\mathrm{e}}}=0, \forall \omega, \\
& \frac{\partial \mathcal{L}}{\partial b_{\omega}}=\mu_{\omega}-\pi_{\omega}^{a r}-\underline{\chi}_{\omega}^{\mathrm{b}}+\bar{\chi}_{\omega}^{\mathrm{b}}=0, \quad \forall \omega, \\
& 0 \leq p^{\mathrm{i}} \perp \underline{\chi}^{\mathrm{p}^{\mathrm{i}}} \geq 0, \\
& 0 \leq \bar{P}^{\mathrm{i}}-p^{\mathrm{i}} \perp \bar{\chi}^{\mathrm{p}^{\mathrm{i}}} \geq 0, \\
& 0 \leq p^{\mathrm{e}} \perp \underline{\chi}^{\mathrm{p}^{\mathrm{e}}} \geq 0, \\
& 0 \leq \bar{P}^{\mathrm{e}}-p^{\mathrm{e}} \perp \bar{\chi}^{\mathrm{p}^{\mathrm{e}}} \geq 0, \\
& 0 \leq \tilde{p}_{\omega}^{\mathrm{i}} \perp \underline{\chi}_{\omega}^{\tilde{\mathrm{p}}^{\mathrm{i}}} \geq 0, \quad \forall \omega, \\
& 0 \leq \bar{P}^{\mathrm{i}}-\tilde{p}_{\omega}^{\mathrm{i}} \perp \bar{\chi}_{\omega}^{\tilde{\mathrm{p}}^{\mathrm{i}}} \geq 0, \quad \forall \omega, \\
& 0 \leq \tilde{p}_{\omega}^{\mathrm{e}} \perp \underline{\chi}_{\omega}^{\tilde{\mathrm{p}}^{\mathrm{e}}} \geq 0, \quad \forall \omega, \\
& 0 \leq \bar{P}^{\mathrm{e}}-\tilde{p}_{\omega}^{\mathrm{e}} \perp \bar{\chi}_{\omega}^{\tilde{\mathrm{p}}^{\mathrm{e}}} \geq 0, \quad \forall \omega, \\
& 0 \leq b_{\omega}+\bar{Y}_{\omega} \perp \underline{\chi}_{\omega}^{\mathrm{b}} \geq 0, \quad \forall \omega, \\
& 0 \leq \bar{Y}_{\omega}-b_{\omega} \perp \bar{\chi}_{\omega}^{\mathrm{b}} \geq 0, \quad \forall \omega .
\end{aligned}
$$

The KKT conditions corresponding to (8) are

$\frac{\partial \mathcal{L}}{\partial \pi_{\omega}^{a r}}=-\left[\left(\tilde{C}^{\mathrm{i}}-\tilde{\lambda}_{\omega}\right) \tilde{p}_{\omega}^{\mathrm{i}}-\left(\tilde{C}^{\mathrm{e}}-\tilde{\lambda}_{\omega}\right) \tilde{p}_{\omega}^{\mathrm{e}}-b_{\omega}\right]+\phi^{a r}$ $-\underline{\chi}_{\omega}^{a r}+\bar{\chi}_{\omega}^{a r}=0, \forall \omega$,

$0 \leq \pi_{\omega}^{a r} \perp \underline{\chi}_{\omega}^{a r} \geq 0, \forall \omega$,

$0 \leq \frac{1}{\alpha^{a r}} \pi_{\omega}^{\Theta}-\pi_{\omega}^{a r} \perp \bar{\chi}_{\omega}^{a r} \geq 0, \forall \omega$,

$\sum_{\omega \in \Omega} \pi_{\omega}^{a r}-1=0$.

The KKT conditions associated with (9) are

$$
\begin{aligned}
& \frac{\partial \mathcal{L}}{\partial \lambda}=\sum_{n \in N} p_{n}-p^{\mathrm{i}}+p^{\mathrm{e}}-\underline{\chi}^{\lambda}+\bar{\chi}^{\lambda}=0, \\
& \frac{\partial \mathcal{L}}{\partial \tilde{\lambda}_{\omega}}=\pi_{\omega}^{\cap}\left(\sum_{n \in N} \tilde{p}_{n \omega}-\tilde{p}_{\omega}^{\mathrm{i}}+\tilde{p}_{\omega}^{\mathrm{e}}\right)-\underline{\chi}_{\omega}^{\tilde{\lambda}}+\bar{\chi}_{\omega}^{\tilde{\lambda}}=0, \forall \omega, \\
& \frac{\partial \mathcal{L}}{\partial \mu_{\omega}}=\sum_{n \in N} a_{n \omega}+b_{\omega}-\underline{\chi}_{\omega}^{\mu}+\bar{\chi}_{\omega}^{\mu}=0, \forall \omega, \\
& 0 \leq \bar{\Lambda}+\lambda \perp \underline{\chi}^{\lambda} \geq 0, \\
& 0 \leq \bar{\Lambda}-\lambda \perp \bar{\chi}^{\lambda} \geq 0, \\
& 0 \leq \bar{\Lambda}+\tilde{\lambda}_{\omega} \perp \underline{\chi}_{\omega}^{\tilde{\lambda}} \geq 0, \forall \omega, \\
& 0 \leq \bar{\Lambda}-\tilde{\lambda}_{\omega} \perp \bar{\chi}_{\omega}^{\tilde{\lambda}} \geq 0, \forall \omega,
\end{aligned}
$$




$$
\begin{aligned}
& 0 \leq \bar{M}+\mu_{\omega} \perp \underline{\chi}_{\omega}^{\mu} \geq 0, \forall \omega, \\
& 0 \leq \bar{M}-\mu_{\omega} \perp \bar{\chi}_{\omega}^{\mu} \geq 0, \forall \omega .
\end{aligned}
$$

Finally, the KKT conditions associated with (10) are

$$
\begin{aligned}
& \frac{\partial \mathcal{L}}{\partial \pi_{\omega}^{\cap}}=-\tilde{\lambda}_{\omega}\left(\sum_{n \in N} \tilde{p}_{n \omega}-\tilde{p}_{\omega}^{\mathrm{i}}+\tilde{p}_{\omega}^{\mathrm{e}}\right)+\phi^{\cap}-\underline{\chi}_{\omega}^{\cap}+\bar{\chi}_{\omega}^{\bigcap} \\
& \quad=0, \forall \omega, \\
& 0 \leq \pi_{\omega}^{\cap} \perp \underline{\chi}_{\omega}^{\cap} \geq 0, \forall \omega, \\
& 0 \leq \frac{1}{\alpha^{\cap}} \pi_{\omega}^{\Theta}-\pi_{\omega}^{\cap} \perp \bar{\chi}_{\omega}^{\cap} \geq 0, \forall \omega, \\
& \sum_{\omega \in \Omega} \pi_{\omega}^{\cap}-1=0 .
\end{aligned}
$$

The resulting mixed complementarity problem is the collection of conditions (15)-(20).

\section{APPENDIX C \\ COMPLETE MARKET FOR RISK}

We solve the risk-averse Nash game with a complete market for risk based on a risk-averse social planner problem, which minimizes the negative expected risk-adjusted system cost as

$$
\begin{aligned}
& \operatorname{Min}_{\Xi} \underbrace{C^{\mathrm{i}} p^{\mathrm{i}}-C^{\mathrm{e}} p^{\mathrm{e}}+\sum_{n \in N} \frac{1}{2} \beta p_{n}^{2}}_{\text {Community cost in the forward market }}-\underbrace{\phi^{\cap}+\frac{1}{\alpha^{\cap}} \sum_{\omega \in \Omega} \pi_{\omega}^{\Theta} \bar{\chi}_{\omega}^{\bigcap}}_{\text {CVaR metric (1c) }} \\
& \text { s.t. }(2 b)-(2 c),(3 b)-(3 d) \text {, } \\
& \phi^{\cap}+\underbrace{\tilde{C}^{\mathrm{i}} \tilde{p}_{\omega}^{\mathrm{i}}-\tilde{C}^{\mathrm{e}} \tilde{p}_{\omega}^{\mathrm{e}}+\sum_{n \in N} \frac{1}{2} \beta \tilde{p}_{n \omega}^{2}}_{\text {Community cost in the spot market }} \leq \bar{\chi}_{\omega}^{\bigcap}: \mu_{\omega}, \forall \omega, \\
& 0 \leq \bar{\chi}_{\omega}^{\bigcap}, \forall \omega,
\end{aligned}
$$

where $\Xi=\left\{p_{n}, p^{\mathrm{i}}, p^{\mathrm{e}}, \tilde{p}_{n \omega}, \tilde{p}_{\omega}^{\mathrm{i}}, \tilde{p}_{\omega}^{\mathrm{e}}, \phi^{\cap}, \bar{\chi}_{\omega}^{\bigcap}\right\}$. The objective function (21a) minimizes the total community cost in the forward market as well as the total expected community cost in the spot market, which are endowed with the CVaR metric introduced in (1c). Variable $\phi^{\cap}$ shows the value-at-risk, and $\bar{\chi}_{\omega}^{\bigcap}$ is a non-negative auxiliary variable. Constraints (21c) and (21d) ensure the non-negativity of CVaR-related variables, where the dual variable $\mu_{\omega}$ of (21c) corresponds to systemwide risk-adjusted probabilities [10], and thus, risk prices.

Given the optimal values obtained for a risk-adjusted social plan $\left(p_{n}, p^{\mathrm{i}}, p^{\mathrm{e}}, \tilde{p}_{n \omega}, \tilde{p}_{\omega}^{\mathrm{i}}, \tilde{p}_{\omega}^{\mathrm{e}}\right)$, as well as risk prices $\mu_{\omega}$, forward $\lambda$ and spot $\tilde{\lambda}_{\omega}$ market-clearing prices, we solve the following optimization problem to derive values for ArrowDebreu securities traded, i.e., $a_{n \omega}$ and $b_{\omega}$ :

$$
\begin{gathered}
\underset{\Phi}{\operatorname{Min}} \sum_{n \in N}(\sum_{\omega \in \Omega} \mu_{\omega}^{*} a_{n \omega}-\underbrace{\phi_{n}^{\rho}+\frac{1}{\alpha_{n}} \sum_{\omega \in \Omega} \pi_{\omega}^{\Theta} \bar{\chi}_{n \omega}^{\rho}}_{\text {Prosumer's CVaR metric (1c) }}) \\
+\sum_{\omega \in \Omega} \mu_{\omega}^{*} b_{\omega}-\underbrace{\phi^{a r}+\frac{1}{\alpha^{a r}} \sum_{\omega \in \Omega} \pi_{\omega}^{\Theta} \bar{\chi}_{\omega}^{a r}}_{\text {Spatial arbitrageur's CVaR metric (1c) }} \\
\text { s.t. } \phi_{n}^{\rho}+\underbrace{\tilde{\lambda}_{\omega}^{*} \tilde{p}_{n \omega}^{*}+\frac{1}{2} \beta \tilde{p}_{n \omega}^{* 2}-a_{n \omega}}_{\text {Prosumer's spot market cost }} \leq \bar{\chi}_{n \omega}^{\rho}, \forall n, \omega,
\end{gathered}
$$

$$
\begin{aligned}
& \phi^{a r}+\underbrace{\left(\tilde{C}^{\mathrm{i}}-\tilde{\lambda}_{\omega}^{*}\right) \tilde{p}_{\omega}^{\mathrm{i}, *}-\left(\tilde{C}^{\mathrm{e}}-\tilde{\lambda}_{\omega}^{*}\right) \tilde{p}_{\omega}^{\mathrm{e}, *}-b_{\omega}}_{\text {Spatial arbitrageur's spot market cost }} \leq \bar{\chi}_{\omega}^{a r}, \forall \omega, \\
& 0 \leq \bar{\chi}_{n \omega}^{\rho}, \forall n, \omega, \\
& 0 \leq \bar{\chi}_{\omega}^{a r}, \forall \omega,
\end{aligned}
$$

where $\Phi=\left\{a_{n \omega}, \phi_{n}^{\rho}, \bar{\chi}_{n \omega}^{\rho}, b_{\omega}, \phi^{a r}, \bar{\chi}_{\omega}^{a r}\right\}$. Parameters denoted by $(\cdot)^{*}$ correspond to values obtained from the risk-averse social planner problem (21). The first line of the objective function (22a) corresponds to Arrow-Debreu security trades by risk-averse prosumers, while the second line refers to the spatial arbitrageur's trades. Constraints (22b) and (22c) define the CVaR metric for each prosumer and the spatial arbitrageur, respectively. Lastly, (22d) and (22e) ensure the non-negativity of CVaR-related variables.

\section{ACKNOWLEDGEMENT}

We would like to thank Harry van der Weijde (University of Edinburgh) for our earlier discussions about risk trading. We also thank David Wozabal and Soner Candas (Technical Universtiy of Munich) for giving feedback on the initial version of the manuscript. We also thank Benjamin F. Hobbs, Puneet Chitkara (Johns Hopkins University), and Christoph Weber (University of Duisburg-Essen) for our discussions after the presentation of this work at the Johns Hopkins University. We also would like to thank Uday Shanbhag (Pennsylvania State University) for his inspiring course on advanced gametheoretic models at the Technical University of Denmark in May 2019. Finally, we would like to thank the three anonymous reviewers for their helpful comments.

\section{REFERENCES}

[1] T. Morstyn, N. Farrell, S. J. Darby, and M. D. McCulloch, "Using peer-to-peer energy-trading platforms to incentivize prosumers to form federated power plants," Nature Energy, vol. 3, pp. 94-101, 2018.

[2] Y. Parag and B. K. Sovacool, "Electricity market design for the prosumer era," Nature Energy, vol. 1, no. 16032, pp. 1-6, 2016.

[3] F. Moret and P. Pinson, "Energy collectives: A community and fairness based approach to future electricity markets," IEEE Trans. Power Syst., vol. 34, no. 5, pp. 3994-4004, 2019.

[4] E. Mengelkamp, J. Gärttner, K. Rock, S. Kessler, L. Orsini, and C. Weinhardt, "Designing microgrid energy markets: A case study: The Brooklyn microgrid," Appl. Energy, vol. 210, pp. 870-880, 2018.

[5] A. P. Shafran, "Learning in games with risky payoffs," Games and Economic Behavior, vol. 75, no. 1, pp. 354-371, 2012.

[6] Y. Zhang and G. B. Giannakis, "Distributed stochastic market clearing with high-penetration wind power," IEEE Trans. Power Syst., vol. 31, no. 2, pp. 895-906, 2016.

[7] B. Willems and J. Morbee, "Market completeness: How options affect hedging and investments in the electricity sector," Energy Econ., vol. 32, no. 4, pp. 786-795, 2010.

[8] R. T. Rockafellar and S. Uryasev, "Conditional value-at-risk for general loss distributions," J. Bank. Financ., vol. 26, no. 7, 2002.

[9] S. A. Gabriel, A. J. Conejo, J. D. Fuller, B. F. Hobbs, and C. Ruiz, Complementarity Modeling in Energy Markets. Springer, 2012.

[10] A. Ehrenmann and Y. Smeers, "Generation capacity expansion in a risky environment: A stochastic equilibrium analysis," Oper. Res., vol. 59, no. 6, pp. 1332-1346, 2011.

[11] J. Kazempour and P. Pinson, "Effects of risk aversion on market outcomes: A stochastic two-stage equilibrium model," Int. Conf. on Prob. Methods Applied to Power Systems (PMAPS 2016), pp. 1-6, 2016, Beijing, China.

[12] R. Cory-Wright and G. Zakeri, "On stochastic auctions in risk-averse electricity markets with uncertain supply," Oper. Res. Lett., vol. 48, no. 3, pp. 376-384, 2020. 
[13] J. Mays, D. P. Morton, and R. P. O'Neill, "Asymmetric risk and fuel neutrality in electricity capacity markets," Nature Energy, vol. 4, pp. 948-956, 2019.

[14] D. Ralph and Y. Smeers, "Risk trading and endogenous probabilities in investment equilibria," SIAM J. Optim., vol. 25, no. 4, pp. 2589$2611,2015$.

[15] G. de Maere d'Aertrycke, A. Ehrenmann, D. Ralph, and Y. Smeers, "Risk trading in capacity equilibrium models," Cambridge Working Paper in Economics, number 1757, Tech. Rep., 2017. [Online]. Available: https://doi.org/10.17863/CAM.17552.

[16] I. Abada, G. de Maere d'Aertrycke, and Y. Smeers, "On the multiplicity of solutions in generation capacity investment models with incomplete markets: A risk-averse stochastic equilibrium approach," Math. Prog., vol. 165, pp. 5-69, 2017.

[17] H. Gérard, V. Leclère, and A. Philpott, "On risk averse competitive equilibrium," Oper. Res. Lett., vol. 46, no. 1, pp. 19-26, 2018.

[18] A. Philpott, M. Ferris, and R. Wets, "Equilibrium, uncertainty and risk in hydro-thermal electricity systems," Math. Prog., vol. 157, pp. 483513, 2016.

[19] F. Moret, P. Pinson, and A. Papakonstantinou, "Heterogeneous risk preferences in community-based electricity markets," Eur. J. Oper. Res., vol. 287, no. 1, pp. 36-48, 2020.

[20] I. Shilov, H. L. Cadre, and A. Busic, "Risk-averse equilibrium analysis and computation," 2020. [Online]. Available: https://arxiv.org/abs/ 2004.02470.

[21] S. Hanif, P. Creutzburg, H. B. Gooi, and T. Hamacher, "Pricing mechanism for flexible loads using distribution grid hedging rights," IEEE Trans. Power Syst., vol. 34, no. 5, pp. 4048-4059, 2019.

[22] Z. Zhang, R. Li, and F. Li, "A novel peer-to-peer local electricity market for joint trading of energy and uncertainty," IEEE Trans. Smart Grid, vol. 11, no. 2, pp. 1205-1215, 2020.

[23] G. De Maere d'Aertrycke, A. Ehrenmann, and Y. Smeers, "Investment with incomplete markets for risk: The need for long-term contracts," Energy Policy, vol. 105, pp. 571-583, 2017.

[24] The Energy Collective Project, https://the-energy-collective-project. com, Accessed: 2020-06-18

[25] EnergyLab Nordhavn - New Urban Energy Infrastructures and Smart Components, http://www.energylabnordhavn.com/, Accessed: 202006-18.

[26] Pebbles Project, https://pebbles-projekt.de/en/, Accessed: 2020-0618.

[27] Renewable Energy Directive, https://data.europa.eu/eli/dir/2018/2001/ oj, Accessed: 2020-06-19.

[28] A. Papavasiliou, "Analysis of distribution locational marginal prices," IEEE Trans. Smart Grid, vol. 9, no. 5, pp. 4872-4882, 2018.

[29] J. Villar, R. Bessa, and M. Matos, "Flexibility products and markets: Literature review," Electr. Power Syst. Res., vol. 154, pp. 329-340, 2018.

[30] K. J. Arrow, "The role of securities in the optimal allocation of riskbearing," The Review of Economic Studies, vol. 31, no. 2, pp. 91-96, 1964.

[31] R. Mieth, M. Roveto, and Y. Dvorkin, "Risk trading in a chanceconstrained stochastic electricity market," IEEE Control Syst. Lett., vol. 5, no. 1, pp. 199-204, 2021.

[32] K. J. Arrow and G. Debreu, "Existence of an equilibrium for a competitive economy," Econometrica, vol. 2, no. 3, pp. 265-290, 1954

[33] P. Artzner, F. Delbaen, J.-M. Eber, and D. Heath, "Coherent measures of risk," Math. Financ., vol. 9, no. 3, pp. 203-228, 1999.

[34] H. Föllmer and A. Schied, "Convex measures of risk and trading constraints," Financ. Stoch., vol. 6, no. 4, pp. 429-447, 2002.

[35] F. Facchinei and J.-S. Pang, Finite-Dimensional Variational Inequalities and Complementarity Problems. Springer, 2007.

[36] V. Dvorkin, J. Kazempour, and P. Pinson, "Electricity market equilibrium under information asymmetry," Oper. Res. Lett., vol. 47, pp. 521-526, 2019.

[37] J. Pang, S. Sen, and U. Shanbhag, "Two-stage non-cooperative games with risk-averse players," Math. Prog., vol. 165, pp. 235-290, 2017.

[38] V. Krebs, L. Schewe, and M. Schmidt, "Uniqueness and multiplicity of market equilibria on DC power flow networks," Eur. J. Oper. Res., vol. 271, no. 1, pp. 165-178, 2018.

[39] F. Murphy, A. Pierru, and Y. Smeers, "Measuring the effects of price controls using mixed complementarity models," Eur. J. Oper. Res., vol. 275, no. 2, pp. 666-676, 2019.

[40] M. C. Ferris and T. S. Munson, "Complementarity problems in GAMS and the PATH solver," J. Econ. Dyn. Control, vol. 24, no. 2, pp. 165$188,2000$.
[41] N. Vespermann, T. Hamacher, and J. Kazempour, "Electronic companion: Risk trading in energy communities," Technical University of Munich, Tech. Rep., 2020. [Online]. Available: https://bitbucket.org/ nivesp/risktrading_energycommunities/.

[42] H. Höschle, H. Le Cadre, Y. Smeers, A. Papavasiliou, and R. Belmans, "An ADMM-based method for computing risk-averse equilibrium in capacity markets," IEEE Trans. Power Syst., vol. 33, no. 5, pp. 48194830, 2018

[43] C. Villani, Optimal Transport: Old and New. Springer, 2008.

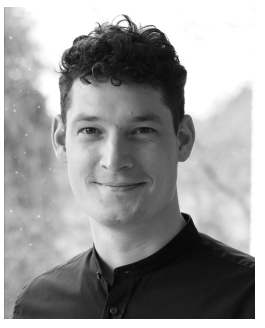

Niklas Vespermann (S'17) received the B.Sc. degree from the RWTH Aachen University, Germany, in 2014, and the M.Sc. degree from the Swiss Federal Institute of Technology, Zurich, Switzerland, in 2016. He is currently pursuing his Ph.D. at the Technical University Munich, Germany. His research interests include power systems, energy markets, game theory, and operations research.

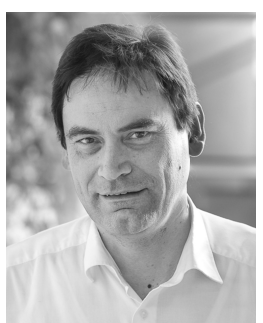

Thomas Hamacher is a full professor in renewable and sustainable energy systems at the Technical University Munich, Germany. His research focuses on energy and systems analysis, focusing on urban energy systems, the integration of renewable energy into the power grid, and innovative nuclear systems including fusion. Other focuses of his work are the methods and fundamentals of energy models.

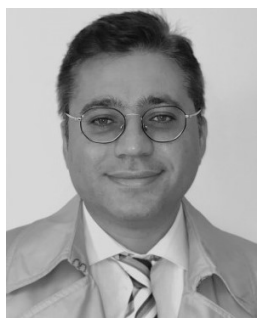

Jalal Kazempour (SM'18) received the Ph.D. degree in electrical engineering from the University of Castilla-La Mancha, Ciudad Real, Spain, in 2013 $\mathrm{He}$ is currently an Associate Professor with the Department of Electrical Engineering, Technical University of Denmark, Kgs. Lyngby, Denmark. His focus area is the intersection of multiple fields, including power and energy systems, electricity markets, optimization, game theory, and machine learning. 